\title{
Material aging causes centrosome weakening and disassembly during mitotic exit
}

Matthäus Mittasch ${ }^{1,3}$, Vanna M. Tran²,3, Manolo U. Rios ${ }^{2}$, Anatol W. Fritsch ${ }^{1}$, Stephen J. Enos $^{1}$, Beatriz Ferreira Gomes ${ }^{1}$, Alec Bond ${ }^{2}$, Moritz Kreysing ${ }^{1,4}$, Jeffrey B. Woodruff ${ }^{2,4}$

1. Max Planck Institute of Molecular Cell Biology and Genetics, Pfotenhauerstrasse 108, 01307 Dresden, Germany

2. Dept. of Cell Biology, Dept. of Biophysics, UT Southwestern Medical Center, Dallas, TX 75390, USA

3. These authors contributed equally

4. For correspondence: kreysing@mpi-cbg.de, jeffrey.woodruff@utsouthwestern.edu

ABSTRACT

Centrosomes must resist microtubule-mediated forces for mitotic chromosome segregation. During mitotic exit, however, centrosomes are deformed and fractured by

17 those same forces, which is a key step in centrosome disassembly. How the functional

18 material properties of centrosomes change throughout the cell cycle, and how they are

19 molecularly tuned remain unknown. Here, we used optically-induced flow perturbations

20 to determine the molecular basis of centrosome strength and ductility in $C$.

21 elegans embryos. We found that both properties declined sharply at anaphase onset,

22 long before natural disassembly. This mechanical transition required PP2A phosphatase

23 and correlated with inactivation of PLK-1 (Polo Kinase) and SPD-2 (Cep192). In vitro,

24 PLK-1 and SPD-2 directly protected centrosome scaffolds from force-induced

25 disassembly. Our results suggest that, prior to anaphase, PLK-1 and SPD-2 confer

26 strength and ductility to the centrosome scaffold so that it can resist microtubule-pulling

27 forces. In anaphase, centrosomes lose PLK-1 and SPD-2 and transition to a weak, brittle

28 state that enables force-mediated centrosome disassembly. 


\section{INTRODUCTION}

Centrosomes nucleate and anchor microtubules that comprise the mitotic spindle, which segregates chromosomes during somatic cell division. Centrosomes are micron-scale, membrane-less organelles containing a structured centriole pair surrounded by an amorphous protein mass called pericentriolar material (PCM). PCM carries out most of the functions of a centrosome, including directing cell polarity, cell migration, and chromosomal segregation (Conduit et al., 2015; Woodruff et al., 2014)

For chromosome segregation, centrosomes must bear microtubule-dependent loads that create tensile stresses. Motor proteins anchored at the plasma membrane attach to and walk along astral microtubules extending from centrosomes. These spatially-fixed motors thus generate cortically-directed pulling forces on centrosomes, and the balance of those forces determines the ultimate position of the mitotic spindle (Colombo et al., 2003; Gonczy et al., 2001; Grill et al., 2001; McNally, 2013; Nguyen-Ngoc et al., 2007). During this time, centrosomes maintain a compact, spherical shape. However, once chromosome segregation is complete and the cell exits mitosis, centrosomes are deformed and fractured by the same microtubule-mediated forces, which is a pronounced event during centrosome disassembly (Megraw et al., 2002; Severson and Bowerman, 2003). How the cell regulates the structural and material integrity of centrosomes is unclear. One possibility is that an increase in cortical forces during mitotic exit induces centrosome disassembly. In C. elegans embryos, the magnitude of microtubule-mediated pulling forces does increase during the metaphase-anaphase transition. Yet, the same increase in pulling forces also occurs in metaphase-arrested embryos without leading to centrosome deformation or fracture (Labbe et al., 2004). Furthermore, artificially increasing pulling forces via csnk-1 RNAi does not cause premature centrosome disassembly (Magescas et al., 2019; Panbianco et al., 2008). These studies suggest that induction of centrosome deformation and fracture during mitotic exit cannot be sufficiently explained by increased microtubule-mediated forces. An alternative hypothesis is that

59 centrosome mechanical properties significantly change to permit force-driven fracture and dispersal during mitotic exit. 
61 PCM provides most of the mass and microtubule nucleation capacity of a centrosome,

62 and it is widely believed to be responsible for bearing microtubule-mediated forces. PCM

63 is dynamic and expands in size and complexity as cells prepare for mitosis. Self-

64 assembly of coiled-coil proteins, such as Cdk5Rap2 (vertebrates), Centrosomin (D.

65 melanogaster) and SPD-5 (C. elegans), creates the underlying structural scaffold of PCM

66 which then recruits "client" proteins that nucleate and regulate microtubules (Conduit et

67 al., 2010; Conduit et al., 2014a; Fong et al., 2008; Hamill et al., 2002; Woodruff et al.,

68 2017; Woodruff et al., 2015). Formation of such micron-scale scaffolds requires additional

69 regulatory clients like Polo Kinase, Aurora A Kinase, and SPD-2/Cep192 (Conduit et al.,

70 2014a; Conduit et al., 2014b; Gomez-Ferreria et al., 2007; Hamill et al., 2002; Hannak et

71 al., 2001; Haren et al., 2009; Lee and Rhee, 2011; Pelletier et al., 2004; Zhu et al., 2008).

72 PCM disassembles at the end of each cell cycle, but the mechanism is not well

73 understood. While this process involves microtubule-mediated PCM fracture and reversal

74 of Polo Kinase phosphorylation (Enos et al., 2018; Magescas et al., 2019; Pimenta-

75 Marques et al., 2016), it remains unclear how PCM fracture is initiated, which key

76 molecular targets are de-phosphorylated, if these activities are linked, and how dynamic

77 material changes might contribute to the disassembly process.

79 A material's load-bearing capacity is determined by its ability to resist permanent 80 deformation and fracture upon stress. In materials science, these properties are 81 described as "strength" and "ductility", respectively. Strength is achieved through high

82 affinity bonding and serves to maintain the material's shape but can sometimes sacrifice

83 flexibility. Ductility is achieved through breakage and reformation of sacrificial bonds or

84 localized neighbor exchange, which dissipates energy over time but sacrifices the

85 material's shape. For example, glass requires large forces to deform, but it cannot deform

86 much before shattering; thus, glass has high strength and low ductility. On the other hand,

87 chewing gum is easily deformed, and it will stretch to great lengths before breaking; thus,

88 gum has low strength and high ductility. Materials with the highest load-bearing capacity

89 are those that combine strength and ductility, such as rubbers, polyampholyte gels, and

90 high-entropy alloys (George et al., 2019; Sun et al., 2013). Over time, these properties 
91 can change via chemical or physical modifications, which is referred to as "material

92 aging".

94 For the centrosome, it remains unexplored how molecular-level interactions between

95 PCM proteins generate meso-level mechanical properties like strength and ductility and

96 how these properties change with time. The non-covalent interactions between PCM

97 scaffold proteins, as well as cross-linking of scaffold molecules by PCM clients, could all

98 contribute. In general, characterizing the mechanical properties of living soft matter-such

99 as cells, organelles, and protein assemblies-has been challenging due to their size (sub-

100 micrometer scale) and low abundance (sub-milligram scale). Techniques like atomic

101 force microscopy and optical trapping can be useful in this respect, but they are limited to

102 easily accessible samples, like the outer membrane of cultured cells and reconstituted

103 protein complexes. As a complementary method to actively probe mechanical properties

104 in cells with limited accessibility, we previously used focused light-induced cytoplasmic

105 streaming (FLUCS)(Mittasch et al., 2018). Specifically, we showed how FLUCS can

106 reveal robust power-law-rheology signatures within the cell cytoplasm, to distinguish

107 between fluid and gel-like states. Fluids undergo unconstrained motion proportional to

108 stimulus time (i.e., they flow), while solids and gels undergo only limited deformations,

109 which stall after small amounts of time due to their intrinsic elastic constraints. As FLUCS

110 functions via thermoviscous flows, which develop independent of the absolute viscosity

111 of the fluid (Weinert et al., 2008), FLUCS is particularly suited to distinguish between

112 highly viscous phases and elastic phases. Such a distinction could not be achieved by

113 passive microrheology, as these two states would exhibit the same fingerprint of reduced

114 motion.

116 The C. elegans embryo is an ideal system to dissect the molecular determinants of PCM

117 load-bearing capacity. First, C. elegans has a limited core set of proteins needed for rapid

118 PCM assembly and disassembly, most of which are conserved across eukaryotes: PLK-

1191 (Polo Kinase homolog), SPD-2 (Cep192 homolog), SPD-5 (functional homolog of

120 Centrosomin and Cdk5Rap2), and LET-92 ${ }^{\text {SUR-6 }}$ (PP2A ${ }^{\text {B55 } \alpha}$ phosphatase homolog)

121 (Decker et al., 2011; Enos et al., 2018; Hamill et al., 2002; Kemp et al., 2004; Magescas 
122 et al., 2019; Pelletier et al., 2004; Schlaitz et al., 2007). Second, it is possible to

123 reconstitute PCM assembly and microtubule nucleation in vitro using purified $C$. elegans

124 proteins (Woodruff et al., 2017; Woodruff et al., 2015). These experiments previously

125 revealed that PCM forms via self-assembly of SPD-5 into spherical, micron-scale

126 scaffolds that recruit PCM client proteins. SPD-2 and PLK-1 enhance SPD-5 self-

127 assembly, while PP2A ${ }^{\text {SUR-6 }}$ removes PLK-1-derived phosphates and promotes PCM

128 disassembly. However, these experiments did not reveal the mechanical properties of the

129 SPD-5 scaffold nor how they are tuned in a cell-cycle-dependent manner.

131 Here, we ask 1) how centrosomes undergo dynamic structural changes to withstand high

132 tensile stresses in mitosis but not during mitotic exit, 2) which mechanical properties are 133 associated with these distinct functional states, and 3) which molecular players and logics

134 regulate the transient existence of centrosomes. To answer these questions, we 135 combined genetics and pharmacological intervention with FLUCS to study the mechanical 136 properties of PCM in C. elegans embryos. Our results revealed that PCM transitions from 137 a strong, ductile state in metaphase to a weak, brittle state in anaphase. This mechanical 138 transition is promoted by PP2A $A^{\text {SUR-6 }}$ and opposed by PLK-1 and SPD-2. Our data suggest 139 that mitotic PCM is a composite of a stable SPD-5 scaffold and proteins that dynamically 140 reinforce the scaffold, such as PLK-1 and SPD-2. During spindle assembly, accumulation 141 of PLK-1 and SPD-2 render the PCM tough enough to resist microtubule-pulling forces.

142 During mitotic exit, departure of PLK-1 and SPD-2 weakens the PCM scaffold to allow 143 microtubule-mediated fracture and disassembly. Thus, PCM undergoes cell-cycle144 regulated material aging that functions to promote its disassembly.

\section{RESULTS}

151 To study the molecular determinants of PCM load-bearing capacity, we studied C. 152 elegans 1-cell embryos, where growth and disassembly of the PCM scaffold is easily 153 visualized using fluorescently-labeled SPD-5 (mMaple::SPD-5)(Figure 1A). During 
154 spindle assembly, the C. elegans PCM scaffold is subject to microtubule-mediated pulling

155 forces, but it maintains its spherical shape and structural integrity. However, during

156 telophase, those same pulling forces deform and fracture the PCM scaffold (Figure

157 1A)(Enos et al., 2018; Severson and Bowerman, 2003). We hypothesized that PCM

158 undergoes an intrinsic mechanical transition from a strong, tough state in metaphase to

159 a weak state in telophase.

We probed PCM mechanical properties using FLUCS-generated flows complemented

162 with fluorescent imaging. Specifically, we equipped a spinning disk confocal microscope

163 with a laser control unit (wavelength $=1455 \mathrm{~nm}$ ) that creates precise, sub-millisecond

164 thermal manipulations (Figure 1B). Unidirectional scans with this laser at $1500 \mathrm{~Hz}$ creates

165 travelling temperature fields that are sufficient to induce flows in a viscous medium,

166 including embryonic cytoplasm (Mittasch et al., 2018)(see Methods). A Peltier-cooled

167 stage insert dissipates excess heat to keep the sample within its physiological

168 temperature range.

We applied FLUCS to C. elegans embryos expressing mMaple::SPD-5 and mCherry-

171 labeled histones (mCherry::H2B). For each experiment, we induced flows crossing the

172 cytoplasm and continuing through the middle of a centrosome, which should apply stress

173 to the PCM scaffold orthogonal to microtubule-derived tensile stresses (Figure 1C).

174 Based on previous experiments (Mittasch et al., 2018), we predicted that cytoplasmic

175 shear flows should weaken the PCM by the relative displacement of scaffold proteins and

176 create a virtual "notch" in the flow path. We used three different amplitudes for the

177 scanning infrared laser $(25,32,40 \mathrm{~mW})$ to generate cytoplasmic flow velocities ranging

178 from 5-20 $\mu \mathrm{m} / \mathrm{min}$ (Figure 1D; Movie S1); these flows scaled quadratically with laser $179 \operatorname{power}\left(R^{2}=0.97\right)$, as one would expect for a predominantly viscous medium (Figure S1A).

180 Simultaneously, we cooled embryos to $17^{\circ} \mathrm{C}$, such that the embryo cytoplasm never 181 exceeded $23^{\circ} \mathrm{C}$ during laser scanning (C. elegans embryos develop properly at any 182 temperature between $16^{\circ} \mathrm{C}$ and $\left.25^{\circ} \mathrm{C}\right)$ (Begasse et al., 2015). 
184 As shown in Figure 1E, FLUCS deformed and fractured mature PCM in anaphase

185 embryos. We also detected a slight increase in cytoplasmic fluorescence surrounding the 186 PCM during FLUCS, indicating that flows can dislodge SPD-5 from the PCM, as predicted

187 (Figure S1B). FLUCS-induced PCM deformation differed starkly between cell cycle 188 stages. FLUCS was not able to visibly deform PCM during metaphase or prior, even 189 though flows were strong enough to detach the centrosome from the spindle and move it 190 toward the cell cortex or out of the focal plane (Figure 1D and S1C and Movies S2, S3; 191 see Metaphase and Prometaphase). On the contrary, FLUCS deformed and eventually 192 fractured PCM during anaphase and telophase (Figure 1E,F; Movie S4,S5); in these 193 experiments, the untreated centrosome remained intact.

195 We then quantified 1) the deformation rate, defined by the rate of PCM length change 196 orthogonal to the flow direction, and 2) the fracture probability, defined by the chance that 197 PCM segments detach completely after FLUCS (Figure 1G; see methods). The PCM 198 deformation rate and fracture probability increased with increasing flow velocity and 199 progression through mitosis (Figure $1 \mathrm{H}, \mathrm{I}$ ). To pinpoint when PCM becomes susceptible 200 to FLUCS-induced deformation, we continuously applied FLUCS to centrosomes starting 201 in metaphase continuing into anaphase. PCM remained spherical and intact during 202 metaphase, but then fractured immediately after anaphase onset, as marked by 203 chromosome segregation (Figure S1D; Movie S6). Our results suggest that PCM 204 resistance to deformation and fracture is high during metaphase, then declines at 205 anaphase onset, $\sim 150$ s prior to full PCM disassembly in telophase. We refer to this 206 change in PCM mechanical properties hereon as the "PCM weakening transition".

208 Since the generation of FLUCS is accompanied by local temperature gradients, we tested 209 if temperature alone affects PCM structure. Bidirectional scanning at $10 \mathrm{kHz}$ creates local 210 temperature gradients without flow, and these conditions did not cause significant PCM 211 deformation or fracture (Figure $1 \mathrm{H}, \mathrm{I}$ and S1E). Thus, centrosome perturbation during 212 FLUCS is primarily due to the flows and not temperature gradients per se. Furthermore, 213 embryos developed properly after cessation of FLUCS (Figure S1F)(Mittasch et al., 
214 2018). We conclude that this established method can be used to study organelles inside

215 a living cell.

217 PCM weakens in anaphase independent of cortical force generation

218 We next wondered if our FLUCS results could be explained by cell-cycle-dependent

219 changes in PCM mechanical properties or changes in cortical force generation. During

220 the metaphase-anaphase transition in early C. elegans embryos, cortical pulling forces

221 increase to induce transverse oscillations and posterior positioning of the mitotic spindle

222 (Pecreaux et al., 2006). Depleting the proteins GPR-1 and GPR-2 (gpr-1/2(RNAi))

223 significantly reduces cortical microtubule-pulling forces and prevents spindle

224 displacement, spindle oscillation, and PCM deformation and fracture (Colombo et al.,

225 2003; Enos et al., 2018; Grill et al., 2003; Magescas et al., 2019; Pecreaux et al., 2006).

226 Thus, we performed FLUCS in gpr-1/2(RNAi) embryos, where we expect only residual

227 cortical forces that remain relatively constant throughout mitosis.

229 In both wild-type and gpr-1/2(RNAi) embryos, FLUCS deformed PCM in anaphase and 230 telophase, but not in metaphase. However, in gpr-1/2(RNAi) embryos, FLUCS-induced

231 PCM deformation rates were slower (Figure 2 A,B; Figure S2A), and FLUCS caused 232 fracturing only in telophase (Figure 2C). These results suggest that PCM mechanical 233 properties change during mitotic exit, but that cortical pulling forces are required to 234 disperse and fracture the pre-weakened PCM scaffold. To further test this idea, we 235 applied $10 \mu \mathrm{g} / \mathrm{ml}$ nocodazole to depolymerize microtubules and performed high-flow 236 FLUCS. Under these conditions, FLUCS did not visibly affect PCM during metaphase, 237 but did dislodge SPD-5 protein from the PCM during telophase (Figure 2D); we did not 238 observe stretching or clean fracture of the PCM during any cell cycle stage. These results 239 indicate that 1) the interactions between SPD-5 scaffold molecules weaken independent 240 of microtubule-pulling forces during mitotic exit, and 2) microtubule-pulling forces are 241 required for stretching and fracture of the PCM during mitotic exit. To test if microtubule242 mediated pulling forces could be sufficient to deform PCM already in metaphase, we 243 depleted a negative regulator of GPR-1/2, casein kinase 1 gamma (csnk-1(RNAi)), which 244 is reported to increase cortical pulling forces $\sim 1.5$-fold (Panbianco et al., 2008). We did 
245 not observe premature deformation or fracture of PCM under these conditions, even

246 though the spindle rocked violently in metaphase (Figure S2B). We conclude that an

247 intrinsic mechanical change in the SPD-5 scaffold is the main driver of PCM weakening

248 during anaphase entry.

250 We next examined the viscoelastic properties of PCM during anaphase by measuring the 251 time-dependent deformation of PCM during FLUCS and relaxation after FLUCS was 252 turned off. We used gpr-1/2(RNAi) embryos to allow residual pulling forces but prevent 253 spindle oscillations, which could complicate our analysis. We observed that continuous 254 application of medium and high-flow FLUCS in gpr-1/2(RNAi) embryos caused time255 dependent strain of the PCM scaffold (Figure 2E). Thus, anaphase PCM is ductile and 256 can experience micron-scale structural rearrangements without complete fracture during 257 stress. Such behavior is seen in viscous materials. When we turned off FLUCS, PCM 258 remained in its strained, elongated state, indicating the absence of a dominant elastic element strong enough to return the PCM to its original shape (Figure $2 \mathrm{E}, \mathrm{F}$ ).

Overall, our FLUCS experiments suggest that the PCM structurally weakens after metaphase. This weakening transition would presumably facilitate PCM disassembly by enabling microtubule-dependent pulling forces to fracture and disperse the PCM scaffold

264 in telophase.

PCM undergoes stepwise compositional changes following anaphase onset

We next investigated the molecular mechanism underlying the PCM weakening transition,

268 in particular, identifying the specific players that determine the dynamic regulation of PCM

269 strength and ductility. PCM is a heterogeneous assembly of proteins required for its 270 assembly and function (Figure 3A). In particular, two critical regulatory proteins, PLK-1 271 (Polo-like Kinase) and SPD-2 (Cep192 homolog), interact with the scaffold protein SPD2725 and enhance its self-assembly into supramolecular structures (Cabral et al., 2019; 273 Decker et al., 2011; Woodruff et al., 2017; Woodruff et al., 2015). PLK-1 and SPD-2, as 274 well as other PCM-localized client proteins, might also reinforce the mature SPD-5 
275 scaffold. On the other hand, loss or inactivation of these proteins could weaken the PCM

276 scaffold.

To analyze PCM composition changes during anaphase, we visualized 9 different GFPlabeled PCM proteins relative to a standard PCM marker, mCherry:: $\gamma$-tubulin. We then measured the integrated density of PCM-localized mCherry and GFP signals during mitosis (Figure 3B). The curves in Figure 3D and Figure S3 represent averages for $>10$ experiments (mean +/- 95\% Cl). mCherry:: $\gamma$-tubulin signal peaked $275-100$ s after anaphase onset, then declined, indicating its departure from PCM. GFP:: $\gamma$-tubulin behaved similarly, as expected (Figure 3C,D and Figure S3). PLK-1 signal decreased immediately after anaphase onset and was no longer detectable $\sim 100$ s later. SPD-2 also departed from the PCM prior to $\gamma$-tubulin. However, the main scaffold protein SPD-5 departed afterward. All other proteins departed coincidentally with $\gamma$-tubulin or soon afterward. TPXL-1 and AIR-1 departed in a biphasic manner: an initial loss of signal occurred prior to $\gamma$-tubulin departure, then a second phase occurred after $\gamma$-tubulin departure. To compare departure kinetics across all experiments, we determined the

291 halfway point of disassembly for each individual GFP and mCherry curve per experiment,

292 then calculated the time differential between halfway points ( $\Delta \mathrm{t}_{\mathrm{EXIT}}$; Figure $\left.3 \mathrm{E}\right)$. The 293 results for anterior and posterior PCM proteins are summarized in Figure 3F and 3G, 294 respectively. A negative $\Delta \mathrm{t}_{\mathrm{EXIT}}$ value indicates GFP::PCM protein departure before $\gamma$ 295 tubulin, and a positive value indicates departure after $\gamma$-tubulin. Our results reveal that 296 PCM composition changes in a stepwise manner during anaphase: PLK-1 departs first,

297 followed by SPD-2, $\gamma$-tubulin, TAC-1, and finally SPD-5 and proteins that form tight 298 complexes with SPD-5 (RSA-1, RSA-2). TPXL-1 and AIR-1 were more variable in their 299 departure, possibly because they localize both to PCM and microtubules that remain after 300 disassembly of the PCM scaffold (Hannak et al., 2001; Ozlu et al., 2005).

Polo Kinase and SPD-2 reinforce the PCM scaffold by increasing its strength and

304 PLK-1 and SPD-2 are the first proteins to depart the PCM during anaphase, when the 
306 the PCM to achieve full strength and stability in metaphase. If this idea is correct, then

307 acute inhibition of PLK-1 phosphorylation or SPD-2 in metaphase might prematurely

308 weaken the PCM, accelerate its disassembly, or reveal hidden material states not

309 previously visible.

311 For acute inhibition of PLK-1, we treated semi-permeable embryos (via perm-1(RNAi))

312 with $10 \mu \mathrm{M}$ Polo Kinase inhibitor Bl-2536 in prometaphase (Carvalho et al., 2011). After

3132 minutes in drug solution, we applied low, medium, and high-flow FLUCS to centrosomes

314 (Figure 4A-C). Under these conditions, low and medium-flow FLUCS deformed

315 metaphase PCM in BI-2536-treated embryos, in contrast to wild-type embryos (Figure

316 4B); in both cases, PCM fracture did not occur. Under high-flow FLUCS, BI2536 treatment

317 increased PCM deformation rate as much as $\sim 11$-fold with fracture occurring only in a

318 minority of the cases (30\%) (Figure 4A,C). The fact that PLK-1 inhibition enabled PCM to

319 be deformed easily without necessarily fracturing suggests that PLK-1 mostly determines

320 PCM strength. This experiment also reveals that wild-type PCM is ductile during

321 metaphase; this could not be observed previously because the deformation resistance of

322 wild-type PCM was too high. BI-2536 treatment also caused premature disassembly of

323 the SPD-5 scaffold in metaphase-arrested embryos, consistent with previous findings

324 (Figure 4D)(Cabral et al., 2019). Our results show that continuous PLK-1 activity is

325 needed for PCM to achieve full strength and maintain integrity until chromosome are

326 separated.

328 Next, we analyzed embryos expressing a temperature-sensitive version of SPD-2 (spd-

329 2(or188ts))(Kemp et al., 2004). We mounted spd-2(or188ts) gfp::spd-5 embryos in cold

330 media and maintained the sample at $17^{\circ} \mathrm{C}$ while imaging until prometaphase, then

331 upshifted the embryos to $25^{\circ} \mathrm{C}$ for $1 \mathrm{~min}$ to inactivate SPD-2or188ts. We then lowered the

332 temperature to $17^{\circ} \mathrm{C}$ to perform FLUCS in metaphase as per usual (Figure 4E)(Note:

333 because of the local heating caused by FLUCS, the treated centrosome remained at

$334 \sim 23^{\circ} \mathrm{C}$ throughout the experiments). The absence of fully functional SPD-2 made PCM

335 more susceptible to FLUCS-induced fracture and disintegration at all applied flow

336 velocities (Figure 4E-G). Even in PCM that did not fracture into observable pieces, the 
337 GFP::SPD-5 signal decayed after application of FLUCS (Figure 4F). We did not observe 338 this phenotype in wild-type PCM or spd-2 mutant PCM not treated with FLUCS (Figure $3394 \mathrm{~F}$ ). Our interpretation of this data is that SPD-2 is required for PCM ductility and strength.

340 Without SPD-2, PCM becomes brittle and susceptible to fracture and diffusion-driven 341 departure of constituents after modest mechanical agitation. In line with this view, 342 inactivation of SPD-2 caused premature disassembly of the SPD-5 scaffold in early 343 anaphase, even without FLUCS perturbations (Figure 4H). Deformation rates were 344 difficult to measure because rapid fracture and vanishing GFP::SPD-5 signal precluded 345 a flow analysis. We conclude that both Polo Kinase and SPD-2 help PCM achieve 346 maximal strength and ductility to prevent disassembly.

348 We next used a minimal in vitro system to test if PLK-1 and SPD-2 directly affect the 349 mechanical properties of the SPD-5 scaffold. When incubated in a crowded environment 350 (e.g. >4\% PEG), purified recombinant SPD-5 assembles into micron-scale condensates 351 that recruit PLK-1, SPD-2, and other PCM proteins (Woodruff et al., 2017). We could not 352 assess SPD-5 condensates using FLUCS because the condensates were propelled 353 quickly away from the flow path (data not shown); thus, our simplified in vitro conditions 354 do not exactly match those found in native cytoplasm. As another way to assess the 355 strength of SPD-5 interactions, we induced disassembly of young RFP-labeled SPD-5 356 condensates (500 nM SPD-5::RFP; 5 min after formation) through application of pipetting 357 shear forces and dilution, then measured the amount of condensates that survived using 358 fluorescence microscopy (Figure 5A)(note: dilution is required to prevent SPD-5 re359 assembly; thus, this assay tests resistance to disassembly only)(Enos et al., 2018). This 360 treatment completely disassembled condensates composed solely of SPD-5 (Figure 361 5B,C). Addition of constitutively active PLK-1 (PLK-1CA; T194D T-loop phospho-mimic) or 362 SPD-2 prevented SPD-5 condensate disassembly, with the combination of the two 363 yielding the greatest protection (Figure 5B,C). Kinase-dead PLK-1 (PLK-1 KD; K67M 364 mutant) did not promote SPD-5 condensate survival. These results suggest that PLK-1 365 phosphorylation of SPD-5, along with direct binding of SPD-2, reinforce the interactions 366 between SPD-5 molecules and thus enhance the ability of PCM to resist disassembly. 
367 Our in vitro and in vivo data together suggest that PLK-1 and SPD-2 tune PCM load-

368 bearing capacity by conferring strength and ductility to the SPD-5 scaffold.

Phosphatase PP2A ${ }^{\text {SUR-6 }}$ promotes PCM disassembly by compromising scaffold ductility

We next investigated how embryos promote the PCM weakening transition during anaphase. PP2A phosphatase localizes to the PCM and plays multiple roles in centriole biogenesis, spindle assembly, and mitotic exit (Wlodarchak and Xing, 2016). The $C$. elegans homolog of PP2A (LET-92) complexed with the B55 $\alpha$ regulatory subunit SUR-6 (PP2A ${ }^{\text {SUR-6 }}$ ) is required for complete PCM disassembly (Enos et al., 2018; Magescas et al., 2019). We thus tested if PP2A ${ }^{\text {SUR-6 }}$ drives PCM disassembly by compromising the

We treated semi-permeable one-cell embryos with $10 \mu \mathrm{M}$ PP2A inhibitor (LB-100) in metaphase, then performed high-flow FLUCS in anaphase. Unlike in wild-type embryos, where PCM fractured quickly after high-flow FLUCS, the PCM in LB-100-treated embryos stretched orthogonal to the induced flow but resisted fracture (Figure 6A). PCM deformation velocity was $\sim 2$-fold higher ( $0.26 \mathrm{vs} .0 .12 \mu \mathrm{m} / \mathrm{min}$ ), initially suggesting that PCM is easier to deform when PP2A is inhibited (Figure 6B). However, PP2A inhibition also elevated the ductility of PCM 1.5-fold (final length divided by the original length) and lowered the fracture probability $>2$-fold in all cell cycle stages (Figure 6C-D). In 2/10 anaphase embryos treated with LB-100, PCM stretched as much as 4-fold in length after FLUCS, reaching up to $10 \mu \mathrm{m}$ while staying connected. PCM was also more resistant to fracture in embryos depleted of the PP2A regulatory subunit SUR-6 (Figure S4A-C).

391 These results suggest that, when PP2A is inhibited, the ductile nature of PCM is 392 preserved throughout anaphase, allowing PCM to absorb more energy overall without 393 fracturing. This is likely achieved through "self-healing", or the breakage and reformation

394 of weak inter-scaffold interactions. The increase in deformation velocity may then result 395 from ductile PCM becoming easier to stretch as it becomes more extended. On the other 396 hand, wild-type PCM is brittle during anaphase and can only be extended short distances 397 before fracturing. We conclude that PP2A normally functions to eliminate "self-healing" 
398 PCM scaffold interactions, thus making PCM brittle during anaphase and susceptible to

399 microtubule-mediated fracture in telophase. Consistent with this conclusion, LB-100

400 inhibition of PP2A or depletion of its regulatory subunit SUR-6 inhibited SPD-5 scaffold

401 disassembly in telophase (Figure 6E) (Enos et al., 2018). We speculate that PCM may

402 be less porous in this mutant ductile state compared to the wild-type brittle state, which

403 could delay PCM disassembly further by preventing access of additional disassembly

404 machinery and/or delaying the departure of PLK-1 and SPD-2. Consistent with the latter

405 concept, both let-92 RNAi and sur-6 RNAi impaired SPD-2 and PLK-1 departure from

406 PCM during anaphase (Figure S4D-G) (Magescas et al., 2019).

408 To determine when and where PP2A might dephosphorylate PCM proteins, we visualized 409 embryos expressing GFP::LET-92, the PP2A catalytic subunit in C. elegans (Schlaitz et 410 al., 2007). GFP::LET-92 localized to the PCM and persisted there until SPD-5 scaffold 411 disassembly, approximately 100s after PLK-1 had departed from the PCM (Figure 6F), 412 consistent with previous observations (Magescas et al., 2019). Our results suggest that, 413 during anaphase, Polo Kinase activity at the PCM ceases and PP2A removes Polo414 delivered phosphates and contributes to SPD-2 departure. This shift in the balance of 415 phosphorylation and dephosphorylation changes the mechanical properties of the PCM, 416 making it more brittle and susceptible to fracture and dissolution.

\section{DISCUSSION}

419 Mitotic spindle assembly and positioning require that centrosomes bear tensile 420 microtubule-dependent forces without structural failure. As mitosis ends, however, these 421 same forces are sufficient to deform and fracture centrosomes, facilitating their 422 disassembly. Disassembly is essential to release centrioles and avoid accumulation of 423 old centrosomes over successive rounds of cell division. Here, we combined flow-driven 424 mechanical perturbations in vivo with biochemical reconstitution in vitro to determine the 425 molecular mechanisms regulating deformation resistance and fracture resistance of 426 PCM, the outer and most massive layer of a centrosome. 
428 PCM mechanical properties, function, and renewal can be achieved through 429 transient reinforcement of the PCM scaffold

430 Using $C$. elegans embryos as a model system, we found that PCM deformation 431 resistance, fracture resistance, and composition are tuned in a cell-cycle-dependent 432 manner (Figure 7A,B). During metaphase, PCM resists both microtubule-mediated forces 433 and induced flow perturbations without deforming or fracturing. In this state, PCM is 434 structured as a reinforced composite, comprising a non-dynamic scaffold of SPD-5 435 molecules filled with a dynamic phase of regulatory molecules like SPD-2 and PLK-1, 436 which frequently bind and unbind the scaffold (Figure 7B). During anaphase, PCM loses 437 PLK-1 and SPD-2 and then becomes susceptible to deformation and fracture. During 438 telophase, PCM is at its weakest and is easily fractured and dispersed by microtubule439 mediated forces, a hallmark step in the PCM disassembly process.

441 Our implementation of flow perturbations in vivo using FLUCS reveals how PLK-1, SPD442 2, and PP2A contribute to the dynamic mechanical properties of the PCM. We interpret 443 deformation resistance as an indicator of strength and fracture resistance and strain as 444 indicators of ductility. Wild-type metaphase PCM is highly resistant to flow perturbations, 445 but underlying features appear in different mutant states (Figure 7C). When PLK-1 is 446 inhibited, the PCM scaffold is easily deformed by FLUCS and stretches without fracturing. 447 Thus, PLK-1 normally maintains PCM strength. On the other hand, when SPD-2 is 448 inhibited, the PCM scaffold is easily fractured and dissolved by FLUCS but does not 449 stretch. Thus, SPD-2 normally maintains PCM ductility and strength. Elimination of either 450 PLK-1 or SPD-2 causes premature PCM disassembly, suggesting that the combination 451 of strength and ductility is necessary for PCM function and maintenance during spindle 452 assembly in metaphase. In anaphase, wild-type PCM is more easily deformed and 453 fractured by FLUCS. Yet, when PP2A is inhibited, PCM is difficult to fracture by FLUCS 454 and instead stretches up to 4 times its original length, revealing that the high ductility of 455 the PCM, which was established prior to metaphase, is preserved. Thus, PP2A normally 456 functions to drive PCM disassembly by reducing PCM ductility. 
458 We propose that that the balance of PLK-1, SPD-2, and PP2A activities determine the 459 mechanical properties and assembly/disassembly state of the PCM (Figure 7D). In 460 metaphase, PLK-1 phosphorylation of SPD-5 and direct binding of SPD-2 reinforce the 461 SPD-5 scaffold, conferring strength and ductility. During anaphase, PLK-1 and SPD-2 462 depart from the PCM, while PP2A phosphatase remains and removes PLK-1-derived 463 phosphates. As a result, PCM becomes progressively brittle and weak, allowing 464 microtubule-dependent forces to deform and fracture it in telophase. Since PLK-1 and 465 SPD-2 stabilize the SPD-5 scaffold, but are themselves dynamic, we call this mode of PCM regulation "transient reinforcement".

Transient reinforcement of the PCM scaffold, in theory, could enable cell-cycle regulated PCM assembly, function, and renewal. In preparation for mitosis, PCM must rapidly assemble and provide a solid foundation for nucleating and anchoring microtubules. If PCM assembly fails, then mitotic spindle assembly and chromosome segregation is severely impaired (Doxsey et al., 1994; Hamill et al., 2002; Sunkel and Glover, 1988). PLK-1 and SPD-2 thus play dual roles in PCM functionality: 1) catalyzing assembly of the PCM scaffold and 2) strengthening it to withstand microtubule-dependent pulling forces (shown here). While PCM is stable during spindle assembly, PCM disassembles in telophase only to be rebuilt in the next cell cycle. PCM disassembly is essential for entry into various post-mitotic states, including the formation of acentriolar oocytes and heart tissue (Pimenta-Marques et al., 2016; Zebrowski et al., 2015). How might transient reinforcement enable PCM disassembly and renewal? Based on in vivo and in vitro FRAP data, PLK-1 and SPD-2 are mobile within the PCM, suggesting that they frequently bind and unbind the SPD-5 scaffold (Laos et al., 2015; Woodruff et al., 2017). Either decreasing their association or increasing their dissociation rates with the SPD-5 scaffold would reduce SPD-2 and PLK-1 levels at the PCM. One potential mechanism is through 484 localized ubiquitination and degradation, which controls Polo Kinase levels at centrosomes in human tissue culture cells during anaphase (Lindon and Pines, 2004). It 486 is currently unknown how PCM levels of SPD-2 or its homolog Cep192 are tuned. The 487 completeness and speed of SPD-2 and PLK-1 removal during anaphase is also 488 suggestive of feedback. Thus, the system could be set up such that minor changes to 
SPD-2 and PLK-1 affinity elicit large, switch-like changes in PCM structure and 490 mechanical properties. Further experiments are needed to define how the other 491 numerous PCM proteins and centriole tethers contribute to PCM mechanical properties.

492 One possible control point is linkage of the SPD-5 scaffold to the centriole via PCMD-1; 493 inactivation of PCMD-1 or laser ablation of centrioles leads to aberrant PCM deformation, 494 presumably because PCM can no longer fully resist microtubule-pulling forces (Cabral et 495 al., 2019; Erpf et al., 2019).

PCM mechanical properties in other eukaryotes

498 The mechanical properties of PCM in other species have yet to be determined. We 499 speculate that our transient reinforcement model for tuning PCM load bearing capacity 500 may be conserved for three reasons. First, diverse eukaryotic species-nematodes, 501 frogs, flies, and human cells-use both Polo Kinase and SPD-2/Cep192 to enhance 502 assembly of the PCM scaffold (Conduit et al., 2014b; Decker et al., 2011; Haren et al., 503 2009; Joukov et al., 2014; Kemp et al., 2004; Pelletier et al., 2004; Woodruff et al., 2015).

504 Second, PP2A is highly conserved in eukaryotes and is required for mitotic exit in these 505 species (Wlodarchak and Xing, 2016). Third, in Drosophila embryos, PCM undergoes cell-cycle-regulated deformation and fracture, termed "flaring", which appears similar to

507 disassembling PCM in C. elegans (Megraw et al., 2002). PCM flares are visible during 508 interphase, cease during metaphase and anaphase, and then escalate during telophase.

509 Flares also require dynamic microtubules. Thus, Drosophila PCM flaring may be due to 510 decreasing PCM strength and ductility during telophase, such that PCM can no longer 511 resist microtubule-mediated forces.

\section{Parallels between PCM and common soft materials in engineering}

514 The mechanical properties and structure of mature PCM are analogous to common 515 composite materials such as flexible plastics and hydrogels. Most modern plastics 516 comprise cross-linked polymer chains embedded with plasticizers, chemicals that make 517 the plastic more flexible and ductile. Over time, these plasticizers exit by diffusion, making 518 the remaining plastic brittle and weak, which is a form of material aging. The rubber sole 519 on a shoe will crack with age; flexibility of the old sole can be restored by impregnating 
520 the rubber with a plasticizer such as silicone. For PCM, the SPD-5 scaffold is most similar

521 to the polymer chains, whereas PLK-1 and SPD-2 could act as plasticizers. Similar to

522 aging rubber losing its plasticizers, our results show that the PCM scaffold becomes brittle

523 and weak during anaphase, coincident with both PLK-1 and SPD-2 leaving the PCM.

PCM is also similar to physical composites like polyampholyte hydrogels, which exhibit a unique combination of high tensile strength and flexibility. Polyampholyte gels comprise polymers cross-linked with a combination of high- and low-affinity non-covalent bonds. Upon stress, the low-affinity bonds break and dissipate energy, while the high-affinity bonds maintain the overall supramolecular structure. The low affinity bonds then quickly reform, resulting in self-healing that prevents structural fatigue from repeated stresses (Sun et al., 2013). These bonds also make the material more ductile, such that it will undergo plastic deformation instead of fracturing. For PCM, it is possible that PLK-1, SPD-2, and other PCM-resident proteins dissipate stress by unbinding from the PCM scaffold, then re-binding to achieve self-healing. Eliminating these weak interactions would make the PCM weaker and more brittle. This would naturally occur in anaphase as PLK-1 and SPD-2 depart from PCM. This concept could also explain why FLUCS induces PCM fracture and deformation in metaphase when we acutely inhibit PLK-1 and SPD-2. Alternatively, in PP2A-inhibited embryos, PCM is ductile due to the preservation of low affinity, self-healing bonds. Although stronger and more ductile than normal, this mutant PCM still weakens and disassembles in telophase, suggesting that another yet unknown process disrupts the strong interactions between SPD-5 molecules.

\section{Conclusion}

544 This work establishes that PCM, the most substantial layer of the centrosome, transitions

545 from a strong, ductile state in metaphase to a weak, brittle state in telophase. This 546 transition is driven by PP2A phosphatase and inactivation of Polo Kinase and SPD-2/Cep547 192, which are essential for centrosome assembly and reinforce the PCM scaffold during 548 metaphase. This mode of mechanical regulation, which we term "transient reinforcement", 549 is a functional form of material aging that allows PCM to resist microtubule-mediated 550 tensile stresses during spindle assembly and then to be fractured and disassembled by 
551 similar forces during mitotic exit. Implicitly, our work demonstrates how flow perturbations

552 can reveal functional mechanical states of membrane-less organelles in vivo.

553

554

555

556

557

558

559

560

561

562

563

564

565

566

567

568

569

570

571

572

573

574

575

576

577

578

579

580

581

\section{FUNDING}

J.B.W. is supported by a Cancer Prevention Research Institute of Texas (CPRIT) grant (RR170063) and the Endowed Scholars program at UT Southwestern. M.K. is supported by the Max Planck Society and ERC grant 853619 , and we acknowledge a DFG-financed DIPP fellowship for M.M.

\section{ACKNOWLEDGEMENTS}

We would like to thank Karen Oegema, Arshad Desai, Anthony Hyman, and Bruce Bowerman for providing strains; Andrea Zinke, Anne Schwager, and Susanne Ernst for help with worm maintenance; Carsten Hoege and David Drechsel for providing the CRISPR reagents; Stephan Daetwyler and Robert Ernst for help with image analysis and script writing; the Live Cell Imaging Facility at UT Southwestern and Light Microscopy Facility at the MPI-CBG for help with microscopy; the Protein Expression Facility at MPICBG for help with protein expression.

\section{AUTHOR CONTRIBUTIONS}

M.M., A.W.F., and M.K. built the FLUCS microscope. M.M. and V.M.T. performed and analyzed the FLUCS experiments on C. elegans embryos. M.U.R. performed the spd-2 temperature-sensitive experiments. B.F-G. and S.J.E. performed two-color imaging of PCM proteins during anaphase and wrote the image analysis scripts. A.B. performed imaging of sur-6(RNAi) mutants. J.B.W. purified proteins and performed the in vitro assays. V.M.T. performed all other in vivo experiments and analysis. J.B.W. and M.K. analyzed data and wrote the manuscript.

\section{FIGURE LEGENDS}

Figure 1. FLUCS reveals changes in PCM deformation resistance and fracture resistance during mitosis in $C$. elegans embryos. 
A. Diagram of mitotic progression in a one-cell C. elegans embryo (top panels). Force generation (arrows) by cortically anchored microtubules aid in chromosome segregation, spindle positioning, and PCM disassembly during telophase. Livecell confocal microscopy images of $C$. elegans embryos expressing a PCM marker (mMaple::SPD-5) and DNA marker (mCherry::HistoneH2B).

B. Diagram of the FLUCS microscope setup (left) and generation of intracellular flows after unidirectional scanning of a $1455 \mathrm{~nm}$ laser at $1500 \mathrm{~Hz}$. Scan path is represented by the magenta line. The magnitudes of local flow velocities are reflected by arrow size.

C. Using FLUCS, flows (red arrows) are generated in the cytoplasm and pass through the PCM (green ball). Microtubule-derived pulling forces (grey arrows) also exert tensile stresses on PCM.

D. Tuning the power of the $1455 \mathrm{~nm}$ laser $(25,32$, and $40 \mathrm{~mW})$ generates three tiers of flow velocity (LOW, MEDIUM, and HIGH). Individual data points are plotted with mean $+/-95 \% \mathrm{Cl} ; \mathrm{n}=5$ measurements per condition.

E. Time lapse images of PCM morphology in anaphase after application of no flow (OFF) or low, medium, and high flow. Orange heading boxes indicate when flow occurs. Arrows indicate flow path and direction. Blue heading boxes indicate when flow is turned off.

F. PCM was subjected to high-flow FLUCS during metaphase, anaphase, and telophase.

G. For each FLUCS experiment, we measured the change in PCM length over time (Deformation rate) and the frequency of complete separations in PCM (Fracture probability).

H. PCM deformation rates were measured in metaphase, anaphase, and telophase using low, medium, and high flow. Individual data points are plotted with mean $+/-$ 95\% Cl; $\mathrm{n}=7,6,7$ (metaphase; LOW, MED, HIGH flow), $\mathrm{n}=7,8,7$ (anaphase), $\mathrm{n}=$ $7,8,9$ (telophase) measurements per condition. $10 \mathrm{kHz}$ bidirectional scanning of the $1455 \mathrm{~nm}$ laser using $40 \mathrm{~mW}$ power, generates heat without producing flows (TEMP control; $\mathrm{n}=4,5,5$ (metaphase, anaphase, telophase)). For high flow, differences are statistically significant using a one-way ANOVA followed by a 
613

Tukey's multiple comparison test (metaphase vs. anaphase, $p=0.04$; metaphase vs. telophase, $p=0.0001$ ).

I. PCM fracture probabilities from experiments in $(H)$. Sample numbers are the same as in $(\mathrm{H})$.

Figure 2. PCM undergoes structural weakening during anaphase, independent of cortical pulling forces.

A. Time-lapse images of $g p r-1 / 2(R N A i)$ embryos treated with $40 \mathrm{~mW}$ FLUCS (high flow).

B. PCM deformation rates in metaphase (M), anaphase (A), and telophase ( $T$ ) using high flow in wild-type and gpr-1/2(RNAi) embryos. Wild-type data are from experiments in Figure 1. Individual data points are plotted with mean $+/-95 \% \mathrm{Cl}$; $\mathrm{n}=7,7,9$ (wild-type; metaphase, anaphase, telophase) and $\mathrm{n}=10,12,14$ (gpr1/2(RNAi)).

C. PCM fracture probabilities from experiments in (B).

D. Permeabilized embryos were treated in metaphase or telophase with $10 \mu \mathrm{g} / \mathrm{ml}$ nocodazole, then subjected to high-flow FLUCS. Representative images are shown on the left, line scans (dotted line in the inset) of fluorescence intensity before and after FLUCS are on the right.

E. Zoomed in time-lapse images of PCM deformation under high-flow FLUCS in a gpr-1/2(RNAi) embryo.

F. Plots comparing PCM length on the long axis orthogonal to flow direction over time. Both high flow and medium flow induce PCM deformation.

\section{Figure 3. Discrete changes in PCM composition correlate with the PCM} weakening transition in anaphase.
A. Diagram of $C$. elegans centrosome architecture and composition.

B. Worm lines were generated that express mCherry-labeled $\gamma$-tubulin (as a standard) and 9 different GFP-labeled PCM proteins (left panels). Fluorescence intensity at the PCM was measured over time (right panels). 
C. Example images from dual-color, time-lapse recording of PCM disassembly in 9 different embryo lines described in (B).

D. Quantification of the experiments in B-C. For each strain, the plots represent the normalized integrated fluorescence density of PCM-localized mCherry-tagged $\gamma$ tubulin compared to the GFP-tagged protein from anaphase onward. Anaphase was indicated by spindle rocking. Shown are the analyses for anterior-localized centrosomes. Data were normalized to the maxima for each individual curve, then these curves were averaged (mean +/- 95\% C.I. $n=13$ ( $\gamma$-tubulin), 9 (PLK-1), 10 (SPD-2), 12 (SPD-5), 14, (RSA-1), 12 (RSA-2), 7 (TAC-1), 10 (TPXL-1), 13 (AIR1)).

E. The order of PCM protein departure was determined by calculating the time lag

Figure 4. Acute inhibition of PLK-1 and SPD-2 induces premature weakening and disassembly of the PCM scaffold.

A. PCM was subjected to high-flow FLUCS during metaphase in wild-type embryos or permeabilized embryos treated with $10 \mu \mathrm{M}$ BI-2536 (inhibitor of Polo Kinases). Permeabilized embryos behaved as wild-type embryos during the first cell division (see methods; Carvalho 2011).

B. PCM deformation rates in metaphase using low, medium, and high flow in wildtype and $\mathrm{Bl}$-2536-treated embryos. Wild-type data are from experiments in Figure 1. Individual data points are plotted showing mean $+/-95 \% \mathrm{Cl} ; \mathrm{n}=6-7$ (wild-type) 
and $n=5-7(B I-2536-$ treated). P-values were calculated using a Mann-Whitney test.

C. PCM fracture probabilities from experiments in (B).

D. Permeabilized embryos were arrested in metaphase using $10 \mu \mathrm{M}$ MG-132, then

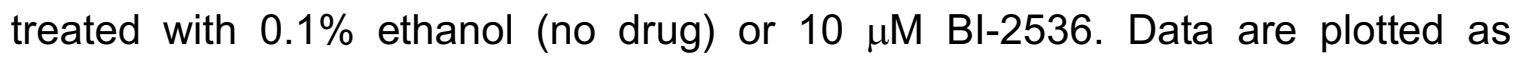
normalized lines representing mean $+/-95 \% \mathrm{Cl} ; n=8$ (no drug) and $n=10$ (BI2536-treated).

E. Embryos expressing GFP::SPD-5 and a temperature-sensitive version of SPD-2 (spd-2(or188ts)) were allowed to assemble centrosomes at the permissive temperature $\left(16^{\circ} \mathrm{C}\right)$, upshifted to the non-permissive temperature $\left(25^{\circ} \mathrm{C}\right)$ for $1 \mathrm{~min}$ during prometaphase, then subjected to high-flow FLUCS during metaphase.

F. For each experiment in spd-2(or188ts) embryos, one centrosome was subjected to FLUCS and the other left alone (control). Integrated fluorescent intensities of the SPD- 5 signal were tracked over time, then normalized to the starting value. Each curve represents a single experiment.

G. PCM fracture probabilities using low, medium, and high flow. Wild-type data are reproduced from Figure 1. $\mathrm{n}=$ 7,7,8 (wild-type) and 5,6,8 (spd-2(or188ts)).

$\mathrm{H}$. Embryos were upshifted from $16^{\circ} \mathrm{C}$ to $23^{\circ} \mathrm{C}$ during metaphase, then imaged during anaphase. Data show integrated fluorescence densities of PCM-localized signal, plotted as normalized lines representing mean $+/-95 \% \mathrm{Cl} ; n=24$ centrosomes in both wild-type and spd-2(or188ts) embryos.

\section{Figure 5. Polo Kinase and SPD-2 protect in vitro reconstituted PCM from induced} disassembly.

A. In vitro SPD-5 condensate disassembly experiment. $500 \mathrm{nM}$ SPD-5::TagRFP was incubated in 9\% PEG to induce spontaneous formation of micron-scale SPD-5 condensates (1. before). After $5 \mathrm{~min}$, the condensates were pipetted harshly and diluted 1:10 in PEG-free buffer, incubated for $10 \mathrm{~min}$, then imaged (2. after).

B. Quantification of total SPD-5 condensate mass per field of view remaining after dilution-induced disassembly. Buffer, $240 \mathrm{nM}$ SPD-2, $500 \mathrm{nM}$ constitutively active PLK-1 (PLK-1 ${ }^{\mathrm{CA}}$ ), and/or $500 \mathrm{nM}$ kinase dead PLK-1 (PLK-1 ${ }^{\mathrm{KD}}$ ) were added the 
beginning. The plot shows total integrated fluorescence density for each field of view (red bars indicate mean +/- 95\% C.I.; $n>22$ images per experiment).

C. Representative images from (B) before and after dilution-induced disassembly.

Figure 6. PCM becomes fracture-resistant and ductile in anaphase after inhibition of PP2A phosphatase.

A. PCM was subjected to high-flow FLUCS during anaphase in wild-type embryos or permeabilized embryos treated with $10 \mu \mathrm{M}$ LB-100 (inhibitor of PP2A phosphatase).

B. PCM deformation rates in anaphase using high flow in wild-type and LB-100treated embryos. Wild-type data are from experiments in Figure 1. Individual data points are plotted with bars representing mean $+/-95 \% \mathrm{Cl} ; \mathrm{n}=7$ (wild-type) and 10 (LB-100-treated) centrosomes. P-values were calculated using a MannWhitney test.

C. Ratio of final PCM length to original length in experiments from (B). Original PCM length was measured before flow began and final PCM length was measured once flow was turned off. P-values were calculated using a Mann-Whitney test.

D. PCM fracture probabilities for high-flow FLUCS in metaphase (M), anaphase (A), and telophase $(T)$. Wild-type data are from experiments in Figure $1 ; n=8,11,11$ (wild-type) and 7,9,6 (LB-100-treated) centrosomes.

E. Permeabilized embryos were treated with no drug or $10 \mu \mathrm{M}$ LB-100 in metaphase, then imaged until 300s after anaphase onset. Data are plotted as normalized lines representing mean +/- 95\% Cl; $n=24$ (no drug) and $n=21$ (LB-100-treated) centrosomes.

F. Dual-color imaging of embryos expressing GFP-tagged LET-92, the PP2A catalytic subunit in C. elegans (GFP.:PP2Ac), and SPD-5::mCherry. Data are plotted as normalized lines representing mean $+/-95 \% \mathrm{Cl} ; \mathrm{n}=10$ centrosomes.

Figure 7. The balance of PLK-1, SPD-2, and PP2A activities tune PCM strength and ductility. 
A. PCM resistance to microtubule-mediated forces peaks in metaphase during spindle assembly, then declines in anaphase and telophase, corresponding to PCM disassembly.

B. PCM-localized levels of 8 different proteins during mitotic progression. In anaphase, PLK-1 levels decline first, followed by SPD-2. The catalytic subunit of PP2A phosphatase (PP2Ac), as well as the main scaffold protein SPD-5, remain at the PCM until late telophase.

C. In metaphase, FLUCS cannot fracture or deform wild-type PCM. However, FLUCS can fracture PCM in spd-2 mutant embryos (i.e., PCM is less ductile) and stretch and deform PCM in PLK-1 inhibited embryos (i.e., PCM is less strong but still ductile). In anaphase, FLUCS easily deforms and fractures wild-type PCM, while it deforms and stretches PCM in PP2A-inhibited embryos (i.e., PCM is more ductile).

D. The combination of PP2A phosphatase activity and the departure of PLK-1 and SPD-2 transitions PCM from a strong, ductile state in metaphase to a weak, brittle state in telophase. This transition enables PCM disassembly and dispersal through

\section{SUPPLEMENTAL FIGURE LEGENDS}

Figure S1. FLUCS control experiments.

A. Power law scaling of cytoplasmic flow with increasing FLUCS laser power. Individual data points represent mean $+/-95 \% \mathrm{Cl} ; \mathrm{n}=5$ embryos per laser condition. Flow velocities were fit with a second-order polynomial.

B. Application of high-flow FLUCS in an anaphase 1-cell embryo. Images are pseudocolored to highlight the subtle increase in cytoplasmic mMaple::SPD-5 fluorescence after flow begins. Note: the centrosome goes out of focus in the first frame when FLUCS begins.

C. Application of high-flow FLUCS in a prometaphase 1-cell embryo. Single plane images are shown. Flow causes the centrosome to leave the plane of focus at $\mathrm{t}=0 \mathrm{~s}$ and $t=20$ s. Flow then displaces the centrosome toward the cortex. 
D. High-flow FLUCS was applied in metaphase, continuing into anaphase (indicated by chromosome segregation at $\mathrm{t}=12 \mathrm{~s}$ ).

E. Example images from the experiment in Figure 1H. Bidirectional scanning of a 40 $\mathrm{mW}$ laser $(1455 \mathrm{~nm})$ at $10 \mathrm{kHz}$ creates local temperature gradients without generating flow.

F. Time-lapse fluorescence and brightfield images of an embryo after cessation of FLUCS. Application of high-flow FLUCS does not affect embryonic development.

Figure S2. Contributions of microtubule pulling forces to PCM deformation.

A. PCM deformation rates in metaphase $(M)$, anaphase $(A)$, and telophase $(T)$ using high flow in wild-type and gpr-1/2(RNAi) embryos or $40 \mathrm{~mW}$ bidirectional laser scanning (temperature control; no flow). Data are from experiments in Figures 1 and 2. Individual data points are plotted with mean $+/-95 \% \mathrm{Cl}$; $\mathrm{n}=$ 7,7,9 (wild-type; metaphase, anaphase, telophase), $\mathrm{n}=10,12,13$ (gpr1/2(RNAi) and $n=4,5,5$ (temperature control). $P$ values were calculated using comparisons tests.

Figure S3. Localization profiles of PCM proteins in the posterior embryo during

B. Time-lapse images of centrosomes in a csnk-1(RNAi) embryo, where microtubule-mediated pulling forces at the cortex are $\sim 1.5$-fold elevated compared to wild type (Panbianco et al., 2008). PCM deformation does not occur prematurely in metaphase. density of PCM-localized mCherry-tagged $\gamma$-tubulin compared to the GFP-tagged protein from anaphase onward. Anaphase was indicated by spindle rocking. Data are normalized to maxima for each individual curve, then averaged; mean $+/-95 \%$ C.I. $n=13$ ( $\gamma$-tubulin), 9 (PLK-1), 10 (SPD-2), 12 (SPD-5), 14, (RSA-1), 12 (RSA2), 7 (TAC-1), 10 (TPXL-1), 13 (AIR-1)). 
Figure S4. FLUCS and localization experiments in sur-6(RNAi) embryos.

A. High-flow FLUCS was applied to a centrosome in an embryo depleted of SUR-6, a PP2A regulatory subunit involved in PCM disassembly.

B. PCM deformation rates in anaphase during high-flow FLUCS in wild-type and sur6(RNAi) embryos. Wild-type data are from experiments in Figure 1. Individual data points are plotted with bars representing mean $+/-95 \% \mathrm{Cl} ; \mathrm{n}=7$ (wild-type) and 10 (sur-6(RNAi)) centrosomes.

C. PCM fracture probabilities in metaphase $(\mathrm{M})$, anaphase $(\mathrm{A})$, and telophase (T) during high-flow FLUCS experiments. $\mathrm{N}=8-11$ (wild-type) and 8-17 (sur-6(RNAi)) centrosomes.

\section{SUPPLEMENTAL MOVIES}

Movie S1. FLUCS flow control using $25 \mathrm{~mW}, 32 \mathrm{~mW}$, and $40 \mathrm{~mW}$ laser scans at 1.5

kHz. Flows were generated in C. elegans 1-cell embryos using three different $1455 \mathrm{~nm}$ laser powers (25 mW, $32 \mathrm{~mW}$, and $40 \mathrm{~mW})$.

Movie S2. High-flow FLUCS targeting the centrosome in a prometaphase 1-cell embryo. Prometaphase $C$. elegans embryos expressing mCherry::histoneH2B (magenta) and GFP::SPD-5 (green) were subjected to high-flow FLUCS (40 mW). Images are of a single confocal plane.

\section{Movie S3. High-flow FLUCS targeting the centrosome in a metaphase 1-cell}

$$
\text { embryo. Metaphase C. elegans embryos expressing mCherry::histoneH2B (magenta) }
$$


826 and GFP::SPD-5 (green) were subjected to high-flow FLUCS (40 mW). Images are of a

827 single confocal plane.

828 Movie S4. High-flow FLUCS targeting the centrosome in an anaphase 1-cell

829 embryo. Anaphase C. elegans embryos expressing mCherry::histoneH2B (magenta)

830 and GFP::SPD-5 (green) were subjected to high-flow FLUCS (40 mW). Images are of a

831 single confocal plane.

832 Movie S5. High-flow FLUCS targeting the centrosome in a telophase 1-cell

833 embryo. Telophase C. elegans embryos expressing mCherry::histoneH2B (magenta)

834 and GFP::SPD-5 (green) were subjected to high-flow FLUCS (40 mW). Images are of a

835 single confocal plane.

836 Movie S6. High-flow FLUCS targeting the centrosome during the metaphase to

837 anaphase transition in a 1-cell embryo. C. elegans embryos expressing

838 mCherry::histoneH2B (magenta) and GFP::SPD-5 (green) were subjected to high-flow

839 FLUCS $(40 \mathrm{~mW})$ during the metaphase-anaphase transition. Images are of a single

840 confocal plane.

841

842 TABLE S1. C. elegans strains used in this study

\begin{tabular}{|l|l|l|l|}
\hline Strain name & genotype & $\begin{array}{l}\text { Creation } \\
\text { method }\end{array}$ & Origin \\
\hline DAM858 & vie11[pAD676; gfp::tac-1]II & CRISPR & $\begin{array}{l}\text { Alexander } \\
\text { Dammermann }\end{array}$ \\
\hline EU584 & spd-2(or188ts) I & mutagenesis & $\begin{array}{l}\text { Bruce } \\
\text { Bowerman }\end{array}$ \\
\hline JWW1 & utsw2[mMaple::spd-5] I & CRISPR & This study \\
\hline JWW13 & $\begin{array}{l}\text { spd-2(or188ts) I; unc-119(ed9) III; ItSi202[pVV103/ } \\
\text { pOD1021; Pspd-2::GFP::SPD-5 RNAiresistant;cb- } \\
\text { unc-119(+)]II }\end{array}$ & $\begin{array}{l}\text { Cross of } \\
\text { EU584 and } \\
\text { OD847 }\end{array}$ & This study \\
\hline JWW35 & $\begin{array}{l}\text { ItSi202[pVV103/ pOD1021; Pspd-2::GFP::SPD-5 } \\
\text { RNAiresistant;cb-unc-119(+)]II ; unc-119(ed3)III; } \\
\text { ddls44[WRM0614cB02 GLCherry::tbg-1;Cbr-unc- } \\
\text { 119(+)] }\end{array}$ & $\begin{array}{l}\text { Cross of } \\
\text { OD847 and } \\
\text { TH169 }\end{array}$ & This study \\
\hline JWW64 & $\begin{array}{l}\text { utsw2[mMaple:::spd-5] I; Itls37 [(pAA64) pie- } \\
\text { 1p::mCherry::his-58+ unc-119(+)] IV. }\end{array}$ & $\begin{array}{l}\text { Cross of } \\
\text { JWW1 and } \\
\text { OD95 }\end{array}$ & This study \\
& $\begin{array}{l}\text { It17[plk-1::gfp+loxP]III ; Itls37 [(pAA64) pie- } \\
\text { 1p::mCherry::his-58 + unc-119(+)] IV; unc-119(ed3) } \\
\text { III }\end{array}$ & $\begin{array}{l}\text { Cross of } \\
\text { OD2425 and } \\
\text { OD95 }\end{array}$ & This study \\
\hline JWW65 & $\begin{array}{l}\text { ItSi203[pVV60; Pspd-2::GFP:::SPD-2 reencoded; } \\
\text { cb-unc-119(+)]II; Itls37 [(pAA64) pie- } \\
\text { 1p:::mCherry::his-58 + unc-119(+)] IV; unc-119(ed3) } \\
\text { III }\end{array}$ & $\begin{array}{l}\text { OD824 and } \\
\text { OD95 }\end{array}$ & This study \\
\hline JWW66 & \multicolumn{2}{|l}{} & \\
\hline
\end{tabular}




\begin{tabular}{|c|c|c|c|}
\hline JWW67 & $\begin{array}{l}\text { unc-119(ed9) III; utsw1[pJWB56; Pspd- } \\
\text { 2::GFP::SPD-5(530E, 627E, 653E, 658E) re- } \\
\text { encoded; cb-unc-119(+)]II }\end{array}$ & $\begin{array}{l}\text { MosSCl, into } \\
\text { EG6699 }\end{array}$ & This study \\
\hline JWW69 & $\begin{array}{l}\text { unc-119(ed9) III; ItSi202[pVV103/ pOD1021; Pspd- } \\
\text { 2::GFP::SPD-5 RNAiresistant;cb-unc-119(+)]Il; } \\
\text { Itls37 [(pAA64) pie-1p::mCherry::his-58 + unc- } \\
\text { 119(+)] IV. }\end{array}$ & $\begin{array}{l}\text { Cross of } \\
\text { OD847 and } \\
\text { OD95 }\end{array}$ & This study \\
\hline JWW70 & $\begin{array}{l}\text { unc-119(ed9) III; utsw1[pJWB56; Pspd- } \\
\text { 2::GFP::SPD-5(530E, 627E, 653E, 658E) re- } \\
\text { encoded; cb-unc-119(+)]II; Itls37 [(pAA64) pie- } \\
\text { 1p::mCherry::his-58 + unc-119(+)] IV. }\end{array}$ & $\begin{array}{l}\text { Cross of } \\
\text { JWW1 and } \\
\text { OD95 }\end{array}$ & This study \\
\hline JWW71 & $\begin{array}{l}\text { It17[plk-1::gfp+loxP]III; unc-119(ed3)III; } \\
\text { ddls44[WRM0614cB02 GLCherry::tbg-1;Cbr-unc- } \\
\text { 119(+)] }\end{array}$ & $\begin{array}{l}\text { Cross of } \\
\text { OD2425 and } \\
\text { TH169 }\end{array}$ & This study \\
\hline JWW72 & $\begin{array}{l}\text { vie11[pAD676; gfp::tac-1]II ; unc-119(ed3)III; } \\
\text { ddls44[WRM0614cB02 GLCherry::tbg-1;Cbr-unc- } \\
\text { 119(+)] }\end{array}$ & $\begin{array}{l}\text { Cross of } \\
\text { DAM858 and } \\
\text { TH169 }\end{array}$ & This study \\
\hline JWW89 & $\begin{array}{l}\text { spd-2(or188ts) I; ItSi202[pVV103/ pOD1021; Pspd- } \\
\text { 2::GFP::SPD-5 RNAiresistant;cb-unc-119(+)]Il; } \\
\text { Itls37 [(pAA64) pie-1p::mCherry::his-58 + unc- } \\
\text { 119(+)] IV. }\end{array}$ & $\begin{array}{l}\text { Cross of } \\
\text { JWW13 and } \\
\text { OD95 }\end{array}$ & This study \\
\hline OD2425 & It17[plk-1::gfp+loxP]III & CRISPR & Karen Oegema \\
\hline OD823 & $\begin{array}{l}\text { ItSi203[pVV60; Pspd-2::GFP::SPD-2 reencoded; } \\
\text { cb-unc-119(+)]II; unc-119(ed3) III }\end{array}$ & $\begin{array}{l}\text { MosSCl, into } \\
\text { EG6699 }\end{array}$ & Karen Oegema \\
\hline OD847 & $\begin{array}{l}\text { unc-119(ed9) III; ItSi202[pVV103/ pOD1021; Pspd- } \\
\text { 2::GFP::SPD-5 RNAiresistant;cb-unc-119(+)]II }\end{array}$ & $\begin{array}{l}\text { MosSCl, into } \\
\text { EG6699 }\end{array}$ & $\begin{array}{l}\text { (Woodruff et al., } \\
\text { 2015) }\end{array}$ \\
\hline OD95 & $\begin{array}{l}\text { unc-119(ed3) III; Itls37 [(pAA64) pie- } \\
\text { 1p::mCherry::his-58 + unc-119(+)] IV; Itls38 [pie- } \\
\text { 1p::GFP::PH(PLC1delta1) + unc-119(+)] }\end{array}$ & $\begin{array}{l}\text { Microparticle } \\
\text { bombardment }\end{array}$ & CGC \\
\hline TH169 & $\begin{array}{l}\text { unc-119(ed3)III; ddls44[WRM0614cB02 } \\
\text { GLCherry::tbg-1;Cbr-unc-119(+)] }\end{array}$ & $\begin{array}{l}\text { Microparticle } \\
\text { bombardment }\end{array}$ & Anthony Hyman \\
\hline TH447 & $\begin{array}{l}\text { unc-119(ed9) III; ddls243[pie-1p::LAP::LET-92; } \\
\text { unc-119(+)]; ddls247[pie-1p::SPD-5(synthetic } \\
\text { introns, CAl 0.65)::mCherry; unc-119(+)] }\end{array}$ & $\begin{array}{l}\text { Microparticle } \\
\text { bombardment }\end{array}$ & Anthony Hyman \\
\hline TH530 & $\begin{array}{l}\text { rsa-1::LAP; unc-119(ed3)III; } \\
\text { ddls44[WRM0614cB02 GLCherry::tbg-1;Cbr-unc- } \\
119(+)]\end{array}$ & $\begin{array}{l}\text { Microparticle } \\
\text { bombardment }\end{array}$ & Anthony Hyman \\
\hline TH531 & $\begin{array}{l}\text { rsa-2::LAP; unc-119(ed3)III; } \\
\text { ddls44[WRM0614cB02 GLCherry::tbg-1;Cbr-unc- } \\
119(+)]\end{array}$ & $\begin{array}{l}\text { Microparticle } \\
\text { bombardment }\end{array}$ & Anthony Hyman \\
\hline TH539 & $\begin{array}{l}\text { spd-2::GFP; unc-119(ed3)III; } \\
\text { ddls44[WRM0614cB02 GLCherry::tbg-1;Cbr-unc- } \\
119(+)]\end{array}$ & $\begin{array}{l}\text { Microparticle } \\
\text { bombardment }\end{array}$ & Anthony Hyman \\
\hline TH571 & $\begin{array}{l}\text { unc-119(ed3)III; ddls12[pie-1p::tpxl-1::GFP;unc- } \\
\text { 119(+)]; ddls44[WRM0614cB02 GLCherry::tbg- } \\
\text { 1;Cbr-unc-119(+)] }\end{array}$ & $\begin{array}{l}\text { Microparticle } \\
\text { bombardment }\end{array}$ & Anthony Hyman \\
\hline TH630 & $\begin{array}{l}\text { ddls44[WRM0614cB02 GLCherry::tbg-1;Cbr-unc- } \\
\text { 119(+)]; ddls62[pie-1p::AIR-1(synthetic introns, CAI } \\
\text { 1.0)::GFP; unc-119(+)]; unc-119(ed3)III }\end{array}$ & $\begin{array}{l}\text { Microparticle } \\
\text { bombardment }\end{array}$ & Anthony Hyman \\
\hline EG6699 & ttTi5605 II; unc-119(ed3) III; oxEx1578. & & CGC \\
\hline
\end{tabular}




\begin{tabular}{|c|c|c|c|c|}
\hline $\begin{array}{l}\text { Plasmid } \\
\text { name }\end{array}$ & Gene & N-term tag & C-term tag & Origin \\
\hline JWV11 & $\begin{array}{l}\text { plk-1(T194D) } \\
\text { constitutively active }\end{array}$ & & PreScission-6xHis & (Woodruff et al., 2015) \\
\hline JWV12 & $\begin{array}{l}\text { plk-1(K67M) } \\
\text { kinase dead }\end{array}$ & & PreScission-6xHis & (Woodruff et al., 2015) \\
\hline JWV2 & $s p d-5(w t)$ & $\begin{array}{l}\text { MBP- } \\
\text { PreScission }\end{array}$ & PreScission-6xHis & (Woodruff et al., 2015) \\
\hline JWV3 & $s p d-5(w t)$ & $\begin{array}{l}\text { MBP- } \\
\text { PreScission }\end{array}$ & $\begin{array}{l}\text { tagRFP- } \\
\text { PreScission-6xHis }\end{array}$ & (Woodruff et al., 2015) \\
\hline JWV6 & $s p d-2(w t)$ & MBP-TEV & TEV-6xHis & (Woodruff et al., 2015) \\
\hline
\end{tabular}

847 TABLE S3. One-way ANOVA and post-hoc tests of anterior PCM disassembly

848 profiles from Figure $3 \mathrm{~F}$

\begin{tabular}{|c|c|c|c|c|}
\hline $\begin{array}{l}\text { Holm-Sidak's } \\
\text { multiple } \\
\text { comparisons test }\end{array}$ & Mean Diff. & Significant? & Summary & $\begin{array}{r}\text { Adjusted P } \\
\text { Value }\end{array}$ \\
\hline AIR-1 vs. TPXL-1 & -25.55 & No & $\mathrm{ns}$ & 0.2725 \\
\hline AIR-1 vs. RSA-1 & -8.310 & No & ns & 0.9615 \\
\hline AIR-1 vs. RSA-2 & -14.38 & No & ns & 0.8174 \\
\hline AIR-1 vs. SPD-2 & 38.26 & Yes & ** & 0.0079 \\
\hline AIR-1 vs. SPD-5 & -24.73 & No & ns & 0.2183 \\
\hline AIR-1 vs. TBG-1 & 8.728 & No & ns & 0.9598 \\
\hline AIR-1 vs. TAC-1 & 0.4412 & No & ns & 0.9963 \\
\hline AIR-1 vs. PLK-1 & 92.29 & Yes & $* * * *$ & $<0.0001$ \\
\hline TPXL-1 vs. RSA-1 & 17.24 & No & ns & 0.8060 \\
\hline TPXL-1 vs. RSA-2 & 11.16 & No & ns & 0.9598 \\
\hline TPXL-1 vs. SPD-2 & 63.81 & Yes & $\star \star \star \star \star *$ & $<0.0001$ \\
\hline TPXL-1 vs. SPD-5 & 0.8171 & No & ns & 0.9963 \\
\hline TPXL-1 vs. TBG-1 & 34.28 & Yes & * & 0.0356 \\
\hline TPXL-1 vs. TAC-1 & 25.99 & No & ns & 0.4307 \\
\hline TPXL-1 vs. PLK-1 & 117.8 & Yes & $\star * * * *$ & $<0.0001$ \\
\hline RSA-1 vs. RSA-2 & -6.074 & No & ns & 0.9615 \\
\hline RSA-1 vs. SPD-2 & 46.57 & Yes & ** & 0.0012 \\
\hline RSA-1 vs. SPD-5 & -16.42 & No & ns & 0.8060 \\
\hline RSA-1 vs. TBG-1 & 17.04 & No & ns & 0.7693 \\
\hline RSA-1 vs. TAC-1 & 8.751 & No & $\mathrm{ns}$ & 0.9615 \\
\hline RSA-1 vs. PLK-1 & 100.6 & Yes & $\star \star * \star *$ & $<0.0001$ \\
\hline RSA-2 vs. SPD-2 & 52.64 & Yes & 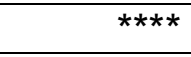 & $<0.0001$ \\
\hline RSA-2 vs. SPD-5 & -10.35 & No & ns & 0.9598 \\
\hline RSA-2 vs. TBG-1 & 23.11 & No & ns & 0.2887 \\
\hline RSA-2 vs. TAC-1 & 14.83 & No & ns & 0.8971 \\
\hline RSA-2 vs. PLK-1 & 106.7 & Yes & $* * * *$ & $<0.0001$ \\
\hline SPD-2 vs. SPD-5 & -62.99 & Yes & $* * * *$ & $<0.0001$ \\
\hline SPD-2 vs. TBG-1 & -29.53 & No & ns & 0.0940 \\
\hline
\end{tabular}




\begin{tabular}{|c|c|c|c|c|}
\hline SPD-2 vs. TAC-1 & -37.82 & Yes & * & 0.0454 \\
\hline SPD-2 vs. PLK-1 & 54.03 & Yes & $* * *$ & 0.0002 \\
\hline SPD-5 vs. TBG-1 & 33.46 & Yes & * & 0.0207 \\
\hline SPD-5 vs. TAC-1 & 25.17 & No & ns & 0.4085 \\
\hline SPD-5 vs. PLK-1 & 117.0 & Yes & $* * * *$ & $<0.0001$ \\
\hline TBG-1 vs. TAC-1 & -8.286 & No & ns & 0.9615 \\
\hline TBG-1 vs. PLK-1 & 83.56 & Yes & $* * * *$ & $<0.0001$ \\
\hline TAC-1 vs. PLK-1 & 91.85 & Yes & $* * * *$ & $<0.0001$ \\
\hline
\end{tabular}

\section{TABLE S4. One-way ANOVA and post-hoc tests of posterior PCM disassembly}

\section{1 profiles from Figure $3 \mathbf{G}$}

\begin{tabular}{|c|c|c|c|c|}
\hline $\begin{array}{l}\text { Holm-Sidak's multiple } \\
\text { comparisons test }\end{array}$ & Mean Diff. & Significant? & Summary & $\begin{array}{r}\text { Adjusted P } \\
\text { Value }\end{array}$ \\
\hline AIR-1 vs. TPXL-1 & -9.235 & No & $\mathrm{ns}$ & 0.9002 \\
\hline AIR-1 vs. RSA-1 & -36.70 & Yes & $* * *$ & 0.0003 \\
\hline AIR-1 vs. RSA-2 & -46.04 & Yes & $\star * * * *$ & $<0.0001$ \\
\hline AIR-1 vs. SPD-2 & 5.014 & No & $\mathrm{ns}$ & 0.9652 \\
\hline AIR-1 vs. SPD-5 & -42.30 & Yes & $* * * *$ & $<0.0001$ \\
\hline AIR-1 vs. TBG-1 & -13.68 & No & $\mathrm{ns}$ & 0.5679 \\
\hline AIR-1 vs. TAC-1 & -34.04 & Yes & ** & 0.0041 \\
\hline AIR-1 vs. PLK-1 & 40.38 & Yes & $\star * * \star *$ & $<0.0001$ \\
\hline TPXL-1 vs. RSA-1 & -27.46 & Yes & * & 0.0334 \\
\hline TPXL-1 vs. RSA-2 & -36.81 & Yes & $* * *$ & 0.0006 \\
\hline TPXL-1 vs. SPD-2 & 14.25 & No & ns & 0.6655 \\
\hline TPXL-1 vs. SPD-5 & -33.07 & Yes & ** & 0.0028 \\
\hline TPXL-1 vs. TBG-1 & -4.446 & No & ns & 0.9652 \\
\hline TPXL-1 vs. TAC-1 & -24.80 & No & ns & 0.1399 \\
\hline TPXL-1 vs. PLK-1 & 49.61 & Yes & $\star * * \star *$ & $<0.0001$ \\
\hline RSA-1 vs. RSA-2 & -9.346 & No & ns & 0.9002 \\
\hline RSA-1 vs. SPD-2 & 41.71 & Yes & $\star * * * *$ & $<0.0001$ \\
\hline RSA-1 vs. SPD-5 & -5.608 & No & $\mathrm{ns}$ & 0.9652 \\
\hline RSA-1 vs. TBG-1 & 23.02 & No & $\mathrm{ns}$ & 0.0790 \\
\hline RSA-1 vs. TAC-1 & 2.661 & No & ns & 0.9652 \\
\hline RSA-1 vs. PLK-1 & 77.07 & Yes & $\star * \star \star *$ & $<0.0001$ \\
\hline RSA-2 vs. SPD-2 & 51.06 & Yes & $\star * * * *$ & $<0.0001$ \\
\hline RSA-2 vs. SPD-5 & 3.739 & No & $\mathrm{ns}$ & 0.9652 \\
\hline RSA-2 vs. TBG-1 & 32.36 & Yes & ${ }^{* *}$ & 0.0014 \\
\hline RSA-2 vs. TAC-1 & 12.01 & No & ns & 0.8399 \\
\hline RSA-2 vs. PLK-1 & 86.42 & Yes & $\star * * \star *$ & $<0.0001$ \\
\hline SPD-2 vs. SPD-5 & -47.32 & Yes & $\star * * \star *$ & $<0.0001$ \\
\hline SPD-2 vs. TBG-1 & -18.70 & No & $\mathrm{ns}$ & 0.2609 \\
\hline SPD-2 vs. TAC-1 & -39.05 & Yes & ${ }^{* *}$ & 0.0014 \\
\hline SPD-2 vs. PLK-1 & 35.36 & Yes & ** & 0.0020 \\
\hline SPD-5 vs. TBG-1 & 28.62 & Yes & ** & 0.0061 \\
\hline SPD-5 vs. TAC- 1 & 8.269 & No & ns & 0.9307 \\
\hline SPD-5 vs. PLK-1 & 82.68 & Yes & $* * * *$ & $<0.0001$ \\
\hline
\end{tabular}




\begin{tabular}{|l|r|r|r|r|}
\hline TBG-1 vs. TAC-1 & -20.35 & No & ns & 0.2689 \\
\hline TBG-1 vs. PLK-1 & 54.06 & Yes & $* * * *$ & $<0.0001$ \\
\hline TAC-1 vs. PLK-1 & 74.41 & Yes & $* * *$ & $<0.0001$ \\
\hline
\end{tabular}

\section{METHODS}

\section{Contact for reagent and resource sharing}

856 Further requests and information for resources and reagents should be directed to and

857 will be fulfilled by the Lead Contact, Jeffrey Woodruff

858 (Jeffrey.woodruff@utsouthwestern.edu).

\section{Experimental model and subject details}

861 C. elegans worm strains were grown on nematode growth media (NGM) plates at 16$86223^{\circ} \mathrm{C}$, following standard protocols (www.wormbook.org). Worm strains used in this study 863 are listed in Table S1 and created using CRISPR (Paix et al., 2015; Paix et al., 2017), 864 MosSCl (Frokjaer-Jensen et al., 2008), or microparticle bombardment. Cas9 enzyme was 865 purified by the Protein Expression Facility at MPI-CBG. For expression of recombinant 866 proteins, we used suspended SF9-ESF S. frugiperda insect cells grown at $27^{\circ} \mathrm{C}$ in ESF 867921 Insect Cell Culture Medium, Protein-Free (Expression Systems), supplemented with 868 Fetal Bovine Serum (2\% final concentration).

\section{RNAi treatment}

871 RNAi was done by feeding using sur-6, gpr-2, csnk-1, and perm-1 feeding clones from

872 the Ahringer and Vidal collections (Source BioScience)(Rual et al., 2004). The spd-5

873 feeding clone targets a region that is reencoded in our MosSCI transgenes (Woodruff et 874 al., 2015). Bacteria were seeded onto nematode growth media (NGM) supplemented 875 with $1 \mathrm{mM}$ isopropyl $\beta$-D-1-thiogalactopyranoside (IPTG) and $100 \mu \mathrm{g} \mathrm{mL}^{-1}$ ampicillin. 876 For perm-1 feeding plates, 0.1 mM IPTG was used. L4 hermaphrodites were grown at $87723^{\circ} \mathrm{C}$ for $24-28$ hours for all conditions except for perm-1, which was at $20^{\circ} \mathrm{C}$ for $18-19$ 878 hours. 
881 For all drug treatments, C. elegans embryos were permeabilized using perm-1 RNAi

882 (Carvalho et al., 2011) and dissected into a 62\% solution of ESF-921 Media (Expression

883 Systems). To arrest the embryos at metaphase, MG-132 (EMD Millipore) was used at 10

$884 \mu \mathrm{M}$ in $62 \% \mathrm{ESF}$, diluted from a $10 \mathrm{mM}$ stock concentration in EtOH. To inhibit PLK-1,

885 Bl-2536 (Advanced ChemBlocks Inc.) was used at $10 \mu \mathrm{M}$, diluted from a $10 \mathrm{mM}$ stock

886 in ethanol. To inhibit PP2A, LB-100 (SelleckChem) was used at $10 \mu \mathrm{M}$, diluted from a

$88710 \mathrm{mM}$ stock in $\mathrm{dH}_{2} \mathrm{O}$. Nocodazole (Sigma) was diluted from a $5 \mathrm{mg} / \mathrm{ml}$ stock

888 concentration in DMSO. Samples were flushed with water or M9 after each experiment

889 to test for permeability (water will cause swelling and M9 will cause shrinking).

\section{Construction of the FLUCS Microscope}

892 To measure the physical material state of centrosomes inside living C. elegans embryos, 893 we performed intracellular flow perturbations by employing the previously published 894 technology FLUCS (Mittasch et al., 2018). The FLUCS setup consisted of three major 895 modules: (i) an infrared laser scanning unit for thermal manipulations, (ii) a microscope 896 allowing to simultaneously induce thermal patterns and to perform high-sensitivity 897 fluorescence imaging, and (iii) a heat management stage.

898 (i) The infrared laser scanning unit consists of a fiber-based infrared Raman laser (CRFL899 20-1455-OM1, 20 Watts, near TEM00 mode profile, Keopsys, France) with a wavelength 900 of $1455 \mathrm{~nm}$, operated in continuous-wave mode and linearly polarized using a polarizing 901 beam splitter cube (CCM1-PBS254, Thorlabs, USA). To precisely correct for the 902 divergence of the laser beam, a telescope was used, composed of two telescope lenses 903 with focal lengths of $f_{2} 1=100 \mathrm{~mm}$ and f_2=150 mm (AC254-C series, Thorlabs, USA), 904 respectively. A lambda-half plate (waveplate, 1/2 1550 Edmund optics, USA) was used 905 to rotate the linearly polarized laser light to match the optical axis of the acoustic-optical 906 deflector (AOD). A variable optical beam expander (4x expander, 36100, Edmund optics, 907 USA) allows control of the beam diameter ( 1.5 mm beam diameter at back-focal-plane 908 was used) without changing the size of the scan pattern. Rapid (up to $1 \mathrm{MHz}$ update rate) 909 and precise (down to $100 \mathrm{~nm}$ ) infrared laser scanning was achieved by utilizing two910 dimensional AOD (AA.DTSXY-A6-145, Pegasus Optik, Germany), electronic oscillators 911 (AA.DRFAIOY-B-0-x, Pegasus Optik, Germany), and electronic amplifiers $2.5 \mathrm{~W}$ 
912 (AA.AMPA-B-34-20.4, Pegasus Optik, Germany). The AOD was controlled by generating 913 analog signals using a custom software in LabVIEW (National instruments, USA) in 914 combination with a PCl controller card (PCle 6369, National Instruments, USA). To

915 precisely translate the AOD-induced beam scanning into the back-focal-plane of the 916 microscope objective lens a telescope composed of two telescope lenses with focal 917 lengths of $f \_3=f \_4=300 \mathrm{~mm}$ (AC254-C series, Thorlabs, USA) was used. A dichroic mirror 918 (F73-705, AHF, Germany) was used to couple the infrared laser beam into the light path 919 of the microscope (IX83, Olympus, Japan), by selectively reflecting the infrared light but 920 transmitting visible wavelengths which were used for fluorescence imaging.

922 (ii) The microscope was equipped with Brightfield (BF) and fluorescent imaging optics. 923 For simultaneous high-resolution fluorescence imaging and precise infrared laser 924 scanning, an infrared-coated microscope objective lens (60x UPLSAPO NA=1.2, W-IR 925 coating, Olympus, Japan) was used, which was operated with heavy water (D2O) as 926 immersion liquid to reduce undesired infrared laser light absorption in the immersion 927 layer. For Brightfield illumination a high-power LED (M565L3, Thorlabs, USA) in 928 combination with dedicated LED driver (LEDD1B, Thorlabs, USA) was used. For confocal 929 fluorescence imaging, a VisiScope confocal imaging system (Visitron, Germany) coupled 930 to a Yokogawa CSU-X1- A12 scan head and an iXON Ultra EMCCD camera (Andor, 931 Ireland) were used.

933 (iii) The heat management unit consisted of a thin sample mounting chamber based on a 934 standard cover slip $(18 \times 18 \times 0.17 \mathrm{~mm})$ (Menzel, Germany) facing the objective lens, and 935 a high thermal conductive sapphire cover slide (thermal conductivity of $27.1 \mathrm{~W} / \mathrm{m} \cdot \mathrm{K}$, 936 SMS-7521, UQG Optics, UK) closing the sandwich-like chamber from the top. To 937 efficiently remove the induced heat from the samples, e.g. C. elegans embryos, the 938 sapphire slide was actively cooled from room temperature to $17^{\circ} \mathrm{C}$. This active cooling 939 was performed by using Peltier elements (TES1-127021, TEC, Conrad) glued to the 940 sapphire slides. The cooling power of the Peltier elements was controlled by a PID 941 hardware controller (TEC-1089-SV, Meerstetter Engineering, Swiss). A custom-built 942 water-cooling stage was used to dissipate the heat produced by the Peltier elements. The 
943 height of the buffer-filled chamber was defined using polystyrene beads (Polybead,

944 Polysciences, Germany) with a diameter of $15 \mu \mathrm{m}$. The height of the resulting chamber

945 was measured by locating the upper and lower chamber surface using a piezo stage.

947 Application of FLUCS within embryos

948 Late L4 hermaphrodites were grown for 18-19 hours on standard NGM or perm-1 feeding 949 plates. Worms were then dissected on an $18 \mathrm{~mm} \times 18 \mathrm{~mm}$ coverslip $(0.17 \mathrm{~mm}$ thickness) 950 in $6 \mu \mathrm{L}$ of M9 buffer or 62\% ESF 921 (for permeabilized embryos) with $15 \mu \mathrm{m}$ polystyrene 951 beads. The sample was placed onto a sapphire microscope slide equipped with Peltier 952 cooling elements, then the coverslip sealed with dental silicone (Picodent twinsil, Picodent, 953 Germany). The cooling stage and sample were then mounted on the FLUCS microscope 954 stage. Embryos were identified and staged using a 10x air objective, then imaged with a 955 60x 1.2 NA Plan Apochromat water immersion objective (Olympus) using $488 \mathrm{~nm}$ and $956561 \mathrm{~nm}$ laser illumination, $1 \mathrm{X} 1$ binning, and 2s intervals.

957 Hydrodynamic flows were generated by scanning the $1455 \mathrm{~nm}$ laser through either 1) 958 center of centrosome or 2) through the cytoplasm for velocity calibration. Custom-written 959 LabVIEW software superimposes the scan path of the infrared laser with the high960 resolution image of the camera. The sub-pixel alignment of the induced flow field and the 961 camera image was verified routinely before the embryonic experiments by using 962 fluorescent tracer particles immersed in a highly viscous sucrose solution. FLUCS 963 experiments used unidirectional but repeated laser scans with $1.5 \mathrm{kHz}$ scan frequency, a 964 scan length of $30 \mu \mathrm{m}$, and three different laser powers (25 mW, $32 \mathrm{~mW}$, and $40 \mathrm{~mW}$ ).

965 Centrosomes were targeted for FLUCS at metaphase, anaphase, or telophase.

966 Centrosomes were affected by FLUCS between 30-60 s. For experiments requiring drug 967 treatment, worms were dissected in $6 \mu \mathrm{L}$ of the specific drug solution and quickly placed 968 on the microscope within 1-2 minutes. To maintain consistency of drug treatment 969 duration, only embryos found exactly at prometaphase (for metaphase experiments) and 970 metaphase (for anaphase experiments) were then targeted for FLUCS. Temperature971 sensitive worms were dissected in cold 62\% ESF-921 media on a cooled dissecting scope 972 and quickly mounted onto the cooling stage, which was maintained at $17^{\circ} \mathrm{C}$. At 
973 prometaphase, temperature was upshifted to $25^{\circ} \mathrm{C}$ for 1 minute, then decreased to $17^{\circ} \mathrm{C}$.

974 Centrosomes were then targeted for FLUCS at metaphase.

975

976

977

978

979

980

981

982

983

984

985

986

987

988

989

990

991

992

993

994

995

996

997

998

999

1000

1001

1002

1003

\section{Confocal microscopy and live-cell imaging}

Adult worms were dissected in M9 before being mounted on a 5\% agar pad for imaging. For live cell imaging with drug treatments, perm-1 adult worms were dissected in 8-10 $\mu \mathrm{L}$ of $62 \%$ ESF 921 with $15 \mu \mathrm{m}$ polystyrene beads (Sigma-Aldrich) on a $22 \times 50 \mathrm{~mm}$ coverslip. Samples were mounted on a $1 \mathrm{~mm}$ thick glass slide with $2 \times 6 \mathrm{~mm}$ laser cut holes $30 \mathrm{~mm}$ apart (Potomac), to produce a flow chamber. In one open chamber, $40 \mu \mathrm{L}$ of the drug solution in $62 \%$ ESF was added during prometaphase. Liquid was wicked from the opposite chamber using a Kimwipe to then allow more of the drug solution to be added to the sample. To arrest embryos in metaphase, perm-1 adult worms were dissected in $10 \mu \mathrm{M}$ MG-132 solution. Cell cycle stage was indicated based on mCherry::HIS-58 fluorescence and cell morphology (metaphase = aligned chromosomes; anaphase $=$ chromosomes separate; telophase $=$ chromosomes de-condense and cytokinetic furrow ingresses).

Time-lapse images were taken using an inverted Nikon Eclipse Ti microscope with a Yokogawa spinning disk confocal head (CSU-X1), piezo Z stage, and an iXon Ultra EMCCD camera (Andor), controlled by Metamorph software. On this system, the $60 \times 1.4$ NA Apochromat oil objective was used to acquire $36 \times 0.5 \mu \mathrm{m}$ Z-stacks every 10 seconds with 100 ms exposures and 2X2 binning. For PCM localization in csnk-1(RNAi) embryos, and PP2A localization, time-lapse images were acquired with an inverted Nikon Eclipse Ti2-E microscope with a Yokogawa confocal scanner unit (CSU-W1), piezo Z stage, and an iXon Ultra 888 EMCCD camera (Andor), controlled by Nikon Elements software. For most experiments, we used a 60x 1.2 NA Plan Apochromat water immersion objective to acquire $35 \times 0.5 \mu \mathrm{m}$ Z-stacks every 10 seconds with $100 \mathrm{~ms}$ exposures and 2X2 binning. Simultaneous imaging with the $488 \mathrm{~nm}$ and $561 \mathrm{~nm}$ lasers was achieved using an OptoSplit II beam splitter (Cairn). For LET-92::GFP imaging, a 100x 1.35 NA Plan Apochromat silicone oil objective was used to acquire $11 \times 0.5 \mu \mathrm{m}$ Z-stacks in 20 second intervals with 100 ms exposures and $2 \times 2$ binning. Images in Figure 3 were taken using 
1004 an inverted Olympus IX81 microscope with a Yokogawa spinning-disk confocal head 1005 (CSU-X1), a 60x 1.2 NA Plan Apochromat water objective, and an iXon EM + DU-897 BV 1006 back illuminated EMCCD (Andor).

1007

1008 spd-2(or188ts) temperature shift assay

1009 JWW69 (control) and JWW89 (spd-2(or188ts)) strains were used for imaging.

1010 Sequencing of JWW89 confirmed a single point mutation in spd-2 resulting in a glycine 1011 to glutamic acid amino acid substitution (G615E) as described in Kemp et. al. Both worm 1012 strains propagated at $16^{\circ} \mathrm{C}$, which is the permissive temperature for spd-2(or188ts). To 1013 prepare the embryos for imaging, a metal block was buried halfway in wet ice. A 24x60 1014 mm glass coverslip (thickness of 1) and a flow chamber slide were placed over the cold 1015 block. To prevent sticking of the glass to the cold block due to water condensation, two 1016 Kimwipes were placed between the glass and the cold block. To minimize exposure to 1017 elevated temperatures during embryo dissection, the glass stage on the dissecting 1018 microscope stand was placed in a $4^{\circ} \mathrm{C}$ fridge and left to cool for approximately $10 \mathrm{~min}$. 1019 Once everything was cold, $10 \mu \mathrm{L}$ of cold M9 plus $15 \mu \mathrm{m}$ polystyrene (Sigma) beads was 1020 pipetted to the middle of the $24 \times 60 \mathrm{~mm}$ cover slip.

1022 For each worm strain, plates were transported inside the ice bucket directly contacting 1023 ice to the dissecting microscope area. The microscope glass stage was taken out from 1024 the fridge and assembled into its place. Three to four adult worms containing a single row 1025 of eggs were transferred to the M9 plus beads on the cover slip still located on top of the 1026 cold block. The coverslip was transferred to the dissecting scope and the worms cut open 1027 using 22G needles. The coverslip was mounted on the flow chamber slide, then the edges 1028 of the cover slip were sealed using clear nail polish. The sample was moved to the 1029 imaging room on the cold block.

1031 The Nikon Eclipse Ti2 microscope described above was used for imaging. Embryos were 1032 staged using a 10X air objective, then imaged with a 60X NA 1.2 water objective. To 1033 rapidly raise the temperature of the sample (up-shift), $40 \mu \mathrm{l}$ of $25^{\circ} \mathrm{C} \mathrm{M9}$ was pipetted into 1034 the flow chamber well. $30 \times 0.5 \mu \mathrm{m} Z$ stacks were collected every $10 \mathrm{~s}$ using simultaneous 
1035 illumination with $488 \mathrm{~nm}$ and $561 \mathrm{~nm}$ lasers (14.7\% and $17.7 \%$ intensity respectively),

$10362 \times 2$ binning, $100 \mathrm{~ms}$ exposures.

\section{Protein expression and purification}

1039 All expression plasmids are listed in Table S2. SPD-5, SPD-2, and PLK-1 proteins were 1040 expressed using the FlexiBAC baculovirus system (Lemaitre et al., 2019) and purified as 1041 previously described (Woodruff and Hyman, 2015; Woodruff et al., 2015), with the 1042 following exception: SPD-2 was stored in its uncleaved form (MBP-TEV-SPD-2).

\section{In vitro SPD-5 condensate disassembly assay}

1045 SPD-5 condensates were formed by diluting $10 \mu \mathrm{M}$ SPD-5 (1:10 mixture of SPD-5 and 1046 SPD-5::TagRFP) in Condensate buffer (25 mM HEPES, pH 7.4, 150 mM KCl) containing 1047 polyethylene glycol 3350 (Sigma) and fresh 0.5 mM DTT. Before use, the SPD-5 stock 1048 solution was centrifuged for $5 \mathrm{~min}$ at $80,000 \mathrm{rpm}$ to remove residual aggregates. $5 \mathrm{~min}$ 1049 after formation, SPD-5 condensates were placed in glass-bottom 96-well dishes (Corning, 1050 4850, high content imaging dish) pre-cleaned with 2\% Hellmanex and washed in water. 1051 For each sample, half was placed in the well undisturbed (control), and the other half was 1052 diluted 10-fold, pipetted 5 times, then placed in a well (induced disassembly). 96-well 1053 plates were imaged on an inverted Nikon Ti-E microscope using a 60x NA 1.4 Plan 1054 Apochromat oil objective, a Zyla cMOS camera (Andor), and MicroManager control 1055 software. For each image, SPD-5 condensates were identified through applying a 1056 threshold then using the particle analyzer function in FIJI. When analyzing condensate 1057 formation, we report the sum of the integrated intensities of each condensate per image 1058 (total condensate mass). Survival \% plotted in Figure 5 assumes a 10-fold loss in total 1059 condensate mass due to dilution.

1061 Quantification and statistical analysis

1062 Images were analyzed with FIJI (https://fiji.sc/), R (https://www.r-project.org/), and 1063 GraphPad Prism (https://www.graphpad.com). For FLUCS experiments, centrosome 1064 deformation was calculated by measuring the long axis (orthogonal to the flow direction) 1065 of PCM-localized SPD-5 at the initial and final time points of PCM deformation, prior to 
1066 fracture (defined below). The deformation rate equaled the difference in PCM lengths

1067 divided by the time interval. Centrosome fracture was measured using line scans across

1068 the long axis of PCM-localized SPD-5. Fracture was scored if signal dropped to

1069 cytoplasmic levels over three consecutive pixels on the long axis across the entire flow

1070 path, and if this signal gap persisted for the rest of the images. For all other experiments,

1071 centrosome tracking and measurement was conducted using max intensity projections,

1072 correction for photobleaching, followed by thresholding and particle analysis. Thresholds

1073 were determined using: mean background intensity of the cytoplasm $+b^{*}$ (standard

1074 deviation of background), where b represents an integer value that is identical for all

1075 samples within an experiment. The integrated fluorescence density for the centrosome-

1076 localized signals were normalized to either the first intensity value or max intensity value

1077 (Figure 3) and plotted over time.

1078

1079 All data are expressed as the mean $\pm 95 \%$ confidence intervals as stated in the figure 1080 legends and results. The value of $\mathrm{n}$ and what $\mathrm{n}$ represents (e.g., number of images,

1081 condensates or experimental replicates) is stated in figure legends and results. Normality

1082 tests were first performed before applying statistical tests. Statistical tests were 1083 performed with GraphPad Prism.

1084

\section{REFERENCES}

1086 Begasse, M.L., M. Leaver, F. Vazquez, S.W. Grill, and A.A. Hyman. 2015. Temperature Dependence of Cell Division Timing Accounts for a Shift in the Thermal Limits of C. elegans and C. briggsae. Cell Rep. 10:647-653. Centrioles in Mitotic Centrosome Growth and Maintenance. Dev Cell. 50:355-366 e356.

Carvalho, A., S.K. Olson, E. Gutierrez, K. Zhang, L.B. Noble, E. Zanin, A. Desai, A. Groisman, and K. Oegema. 2011. Acute drug treatment in the early C. elegans embryo. PLoS One. 6:e24656.

Colombo, K., S.W. Grill, R.J. Kimple, F.S. Willard, D.P. Siderovski, and P. Gonczy. 2003. Translation of polarity cues into asymmetric spindle positioning in Caenorhabditis elegans embryos. Science. 300:1957-1961.

Conduit, P.T., K. Brunk, J. Dobbelaere, C.I. Dix, E.P. Lucas, and J.W. Raff. 2010. Centrioles regulate centrosome size by controlling the rate of $\mathrm{Cnn}$ incorporation into the PCM. Current biology : CB. 20:2178-2186. 
Conduit, P.T., Z. Feng, J.H. Richens, J. Baumbach, A. Wainman, S.D. Bakshi, J. Dobbelaere, S. Johnson, S.M. Lea, and J.W. Raff. 2014a. The centrosome-specific phosphorylation of Cnn by Polo/PIk1 drives Cnn scaffold assembly and centrosome maturation. Dev Cell. 28:659-669.

Conduit, P.T., J.H. Richens, A. Wainman, J. Holder, C.C. Vicente, M.B. Pratt, C.I. Dix, Z.A. Novak, I.M. Dobbie, L. Schermelleh, and J.W. Raff. 2014b. A molecular mechanism of mitotic centrosome assembly in Drosophila. eLife. 3:e03399.

Conduit, P.T., A. Wainman, and J.W. Raff. 2015. Centrosome function and assembly in animal cells. Nature reviews. Molecular cell biology. 16:611-624.

Decker, M., S. Jaensch, A. Pozniakovsky, A. Zinke, K.F. O'Connell, W. Zachariae, E. Myers, and A.A. Hyman. 2011. Limiting amounts of centrosome material set centrosome size in C. elegans embryos. Current biology: CB. 21:1259-1267.

Doxsey, S.J., P. Stein, L. Evans, P.D. Calarco, and M. Kirschner. 1994. Pericentrin, a highly conserved centrosome protein involved in microtubule organization. Cell. 76:639-650.

Enos, S.J., M. Dressler, B.F. Gomes, A.A. Hyman, and J.B. Woodruff. 2018. Phosphatase PP2A and microtubule-mediated pulling forces disassemble centrosomes during mitotic exit. Biol Open. 7.

Erpf, A.C., L. Stenzel, N. Memar, M. Antoniolli, M. Osepashvili, R. Schnabel, B. Conradt, and T. Mikeladze-Dvali. 2019. PCMD-1 Organizes Centrosome Matrix Assembly in C. elegans. Current biology : CB. 29:1324-1336 e1326.

Fong, K.W., Y.K. Choi, J.B. Rattner, and R.Z. Qi. 2008. CDK5RAP2 is a pericentriolar protein that functions in centrosomal attachment of the gamma-tubulin ring complex. Mol Biol Cell. 19:115-125.

Frokjaer-Jensen, C., M.W. Davis, C.E. Hopkins, B.J. Newman, J.M. Thummel, S.P. Olesen, M. Grunnet, and E.M. Jorgensen. 2008. Single-copy insertion of transgenes in Caenorhabditis elegans. Nat Genet. 40:1375-1383.

George, E.P., D. Raabe, and R.O. Ritchie. 2019. High-entropy alloys. Nature Reviews Materials. Gomez-Ferreria, M.A., U. Rath, D.W. Buster, S.K. Chanda, J.S. Caldwell, D.R. Rines, and D.J. Sharp. 2007. Human Cep192 is required for mitotic centrosome and spindle assembly. Current biology : CB. 17:1960-1966.

Gonczy, P., S. Grill, E.H. Stelzer, M. Kirkham, and A.A. Hyman. 2001. Spindle positioning during the asymmetric first cell division of Caenorhabditis elegans embryos. Novartis Found Symp. 237:164-175; discussion 176-181.

Grill, S.W., P. Gonczy, E.H. Stelzer, and A.A. Hyman. 2001. Polarity controls forces governing asymmetric spindle positioning in the Caenorhabditis elegans embryo. Nature. 409:630633.

Grill, S.W., J. Howard, E. Schaffer, E.H. Stelzer, and A.A. Hyman. 2003. The distribution of active force generators controls mitotic spindle position. Science. 301:518-521.

Hamill, D.R., A.F. Severson, J.C. Carter, and B. Bowerman. 2002. Centrosome maturation and mitotic spindle assembly in C. elegans require SPD-5, a protein with multiple coiled-coil domains. Dev Cell. 3:673-684.

Hannak, E., M. Kirkham, A.A. Hyman, and K. Oegema. 2001. Aurora-A kinase is required for centrosome maturation in Caenorhabditis elegans. J Cell Biol. 155:1109-1116. 
1143 Harashima, H., N. Dissmeyer, and A. Schnittger. 2013. Cell cycle control across the eukaryotic kingdom. Trends Cell Biol. 23:345-356.

Haren, L., T. Stearns, and J. Luders. 2009. PIk1-dependent recruitment of gamma-tubulin complexes to mitotic centrosomes involves multiple PCM components. PLoS One. 4:e5976.

Joukov, V., J.C. Walter, and A. De Nicolo. 2014. The Cep192-organized aurora A-PIk1 cascade is essential for centrosome cycle and bipolar spindle assembly. Mol Cell. 55:578-591.

Kemp, C.A., K.R. Kopish, P. Zipperlen, J. Ahringer, and K.F. O'Connell. 2004. Centrosome maturation and duplication in C. elegans require the coiled-coil protein SPD-2. Dev Cell. 6:511-523.

Labbe, J.C., E.K. McCarthy, and B. Goldstein. 2004. The forces that position a mitotic spindle asymmetrically are tethered until after the time of spindle assembly. J Cell Biol. 167:245256.

Laos, T., G. Cabral, and A. Dammermann. 2015. Isotropic incorporation of SPD-5 underlies centrosome assembly in C. elegans. Current biology : CB. 25:R648-649.

Lee, K., and K. Rhee. 2011. PLK1 phosphorylation of pericentrin initiates centrosome maturation at the onset of mitosis. J Cell Biol. 195:1093-1101.

Lemaitre, R.P., A. Bogdanova, B. Borgonovo, J.B. Woodruff, and D.N. Drechsel. 2019. FlexiBAC: a versatile, open-source baculovirus vector system for protein expression, secretion, and proteolytic processing. BMC Biotechnol. 19:20.

Lindon, C., and J. Pines. 2004. Ordered proteolysis in anaphase inactivates Plk1 to contribute to proper mitotic exit in human cells. J Cell Biol. 164:233-241.

Magescas, J., J.C. Zonka, and J.L. Feldman. 2019. A two-step mechanism for the inactivation of microtubule organizing center function at the centrosome. eLife. 8.

McNally, F.J. 2013. Mechanisms of spindle positioning. J Cell Biol. 200:131-140.

Megraw, T.L., S. Kilaru, F.R. Turner, and T.C. Kaufman. 2002. The centrosome is a dynamic structure that ejects PCM flares. J Cell Sci. 115:4707-4718.

Mittasch, M., P. Gross, M. Nestler, A.W. Fritsch, C. Iserman, M. Kar, M. Munder, A. Voigt, S. Alberti, S.W. Grill, and M. Kreysing. 2018. Non-invasive perturbations of intracellular flow reveal physical principles of cell organization. Nat Cell Biol. 20:344-351.

Nguyen-Ngoc, T., K. Afshar, and P. Gonczy. 2007. Coupling of cortical dynein and G alpha proteins mediates spindle positioning in Caenorhabditis elegans. Nat Cell Biol. 9:12941302.

Ozlu, N., M. Srayko, K. Kinoshita, B. Habermann, T. O'Toole E, T. Muller-Reichert, N. Schmalz, A. Desai, and A.A. Hyman. 2005. An essential function of the C. elegans ortholog of TPX2 is to localize activated aurora A kinase to mitotic spindles. Dev Cell. 9:237-248.

Paix, A., A. Folkmann, D. Rasoloson, and G. Seydoux. 2015. High Efficiency, Homology-Directed Genome Editing in Caenorhabditis elegans Using CRISPR-Cas9 Ribonucleoprotein Complexes. Genetics. 201:47-54.

Paix, A., A. Folkmann, and G. Seydoux. 2017. Precision genome editing using CRISPR-Cas9 and linear repair templates in C. elegans. Methods. 121-122:86-93.

Panbianco, C., D. Weinkove, E. Zanin, D. Jones, N. Divecha, M. Gotta, and J. Ahringer. 2008. A casein kinase 1 and PAR proteins regulate asymmetry of a PIP(2) synthesis enzyme for asymmetric spindle positioning. Dev Cell. 15:198-208. 
Pecreaux, J., J.C. Roper, K. Kruse, F. Julicher, A.A. Hyman, S.W. Grill, and J. Howard. 2006. Spindle oscillations during asymmetric cell division require a threshold number of active cortical force generators. Current biology : CB. 16:2111-2122.

Pelletier, L., N. Ozlu, E. Hannak, C. Cowan, B. Habermann, M. Ruer, T. Muller-Reichert, and A.A. Hyman. 2004. The Caenorhabditis elegans centrosomal protein SPD-2 is required for both pericentriolar material recruitment and centriole duplication. Current biology : $C B$. 14:863-873.

Pimenta-Marques, A., I. Bento, C.A. Lopes, P. Duarte, S.C. Jana, and M. Bettencourt-Dias. 2016. A mechanism for the elimination of the female gamete centrosome in Drosophila melanogaster. Science. 353:aaf4866.

Rual, J.F., J. Ceron, J. Koreth, T. Hao, A.S. Nicot, T. Hirozane-Kishikawa, J. Vandenhaute, S.H. Orkin, D.E. Hill, S. van den Heuvel, and M. Vidal. 2004. Toward improving Caenorhabditis elegans phenome mapping with an ORFeome-based RNAi library. Genome Res. 14:21622168.

Schlaitz, A.L., M. Srayko, A. Dammermann, S. Quintin, N. Wielsch, I. MacLeod, Q. de Robillard, A. Zinke, J.R. Yates, 3rd, T. Muller-Reichert, A. Shevchenko, K. Oegema, and A.A. Hyman. 2007. The $C$. elegans RSA complex localizes protein phosphatase $2 A$ to centrosomes and regulates mitotic spindle assembly. Cell. 128:115-127.

Severson, A.F., and B. Bowerman. 2003. Myosin and the PAR proteins polarize microfilamentdependent forces that shape and position mitotic spindles in Caenorhabditis elegans. $J$ Cell Biol. 161:21-26.

Sun, T.L., T. Kurokawa, S. Kuroda, A.B. Ihsan, T. Akasaki, K. Sato, M.A. Haque, T. Nakajima, and J.P. Gong. 2013. Physical hydrogels composed of polyampholytes demonstrate high toughness and viscoelasticity. Nat Mater. 12:932-937.

Sunkel, C.E., and D.M. Glover. 1988. polo, a mitotic mutant of Drosophila displaying abnormal spindle poles. J Cell Sci. 89 ( Pt 1):25-38.

Weinert, F.M., J.A. Kraus, T. Franosch, and D. Braun. 2008. Microscale fluid flow induced by thermoviscous expansion along a traveling wave. Phys Rev Lett. 100:164501.

Wlodarchak, N., and Y. Xing. 2016. PP2A as a master regulator of the cell cycle. Crit Rev Biochem Mol Biol. 51:162-184.

Woodruff, J.B., B. Ferreira Gomes, P.O. Widlund, J. Mahamid, A. Honigmann, and A.A. Hyman. 2017. The Centrosome Is a Selective Condensate that Nucleates Microtubules by Concentrating Tubulin. Cell. 169:1066-1077 e1010.

Woodruff, J.B., and A.A. Hyman. 2015. Method: In vitro analysis of pericentriolar material assembly. Methods Cell Biol. 129:369-382.

Woodruff, J.B., O. Wueseke, and A.A. Hyman. 2014. Pericentriolar material structure and dynamics. Philos Trans R Soc Lond B Biol Sci. 369.

Woodruff, J.B., O. Wueseke, V. Viscardi, J. Mahamid, S.D. Ochoa, J. Bunkenborg, P.O. Widlund, A. Pozniakovsky, E. Zanin, S. Bahmanyar, A. Zinke, S.H. Hong, M. Decker, W. Baumeister, J.S. Andersen, K. Oegema, and A.A. Hyman. 2015. Centrosomes. Regulated assembly of a supramolecular centrosome scaffold in vitro. Science. 348:808-812.

Zebrowski, D.C., S. Vergarajauregui, C.C. Wu, T. Piatkowski, R. Becker, M. Leone, S. Hirth, F. Ricciardi, N. Falk, A. Giessl, S. Just, T. Braun, G. Weidinger, and F.B. Engel. 2015. 
bioRxiv preprint doi: https://doi.org/10.1101/866434; this version posted December 5, 2019. The copyright holder for this preprint (which was not certified by peer review) is the author/funder, who has granted bioRxiv a license to display the preprint in perpetuity. It is made available under aCC-BY-NC-ND 4.0 International license.

Developmental alterations in centrosome integrity contribute to the post-mitotic state of mammalian cardiomyocytes. eLife. 4.

1232 Zhu, F., S. Lawo, A. Bird, D. Pinchev, A. Ralph, C. Richter, T. Muller-Reichert, R. Kittler, A.A. Hyman, and L. Pelletier. 2008. The mammalian SPD-2 ortholog Cep192 regulates centrosome biogenesis. Current biology : CB. 18:136-141. 


\section{Mittasch et al. Figure 1}

A

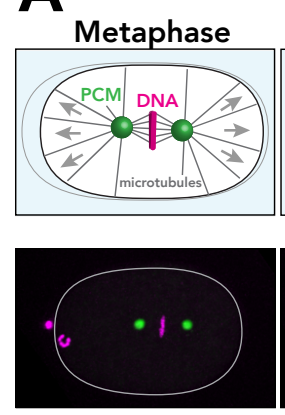

C

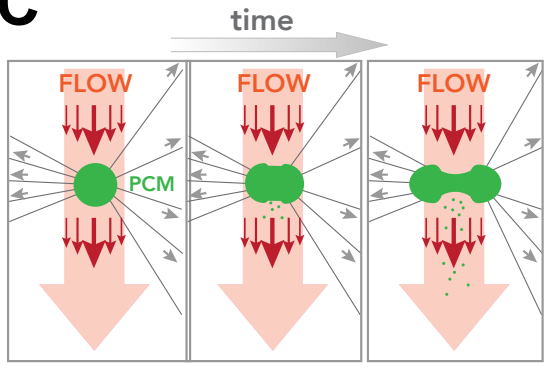

$\rightarrow$ tensile stress from microtubules

$\mathbf{F}$
B
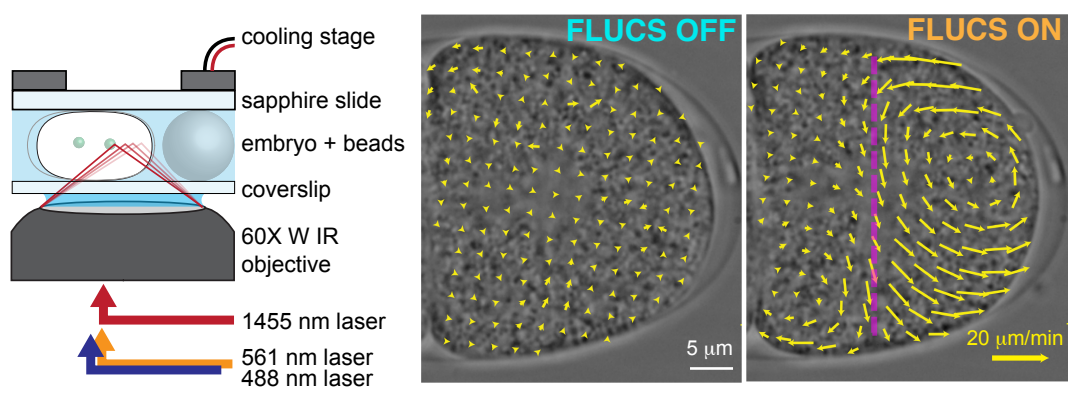

E Anaphase

mMaple::SPD-5

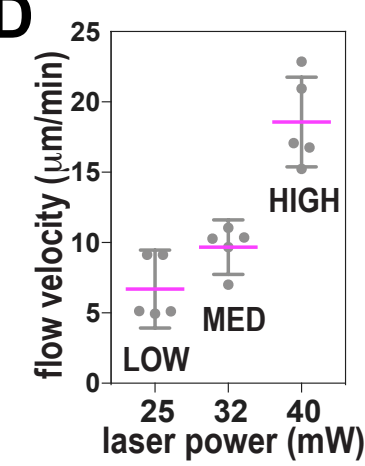

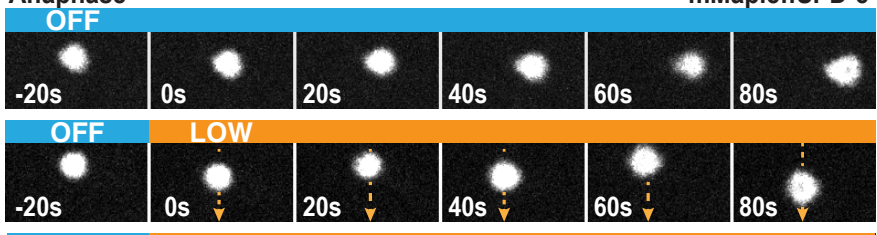

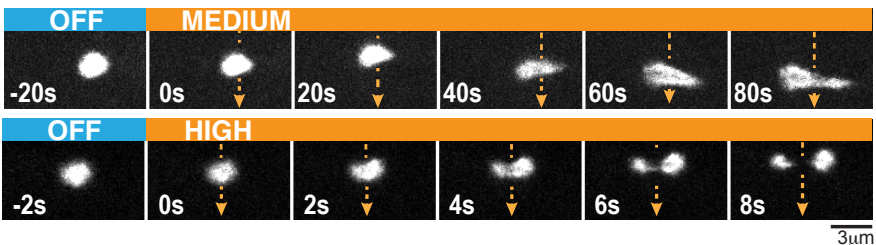

Metaphase

mMaple::SPD-5 mCherry::H2B

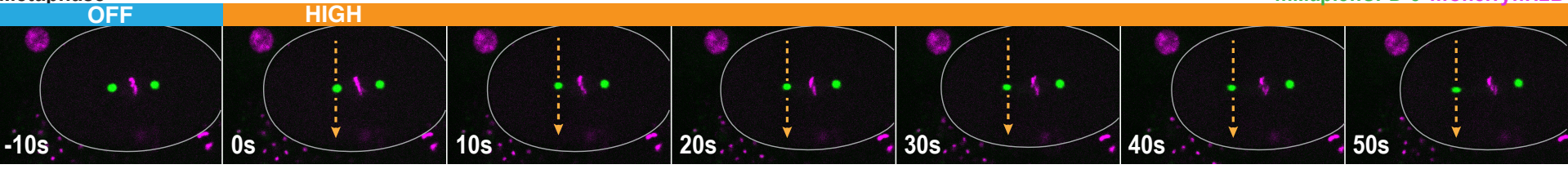

Anaphase

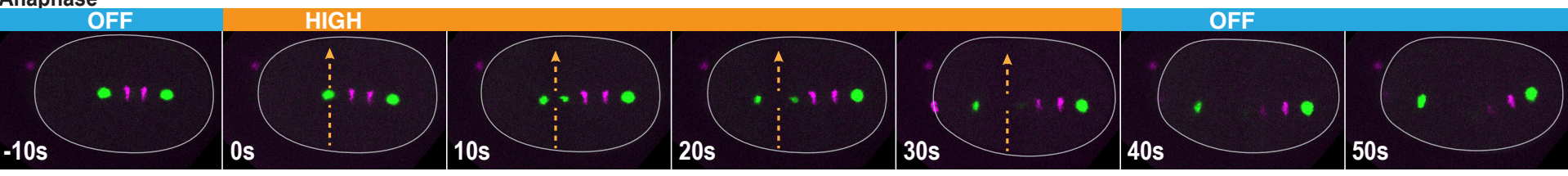

Telophase

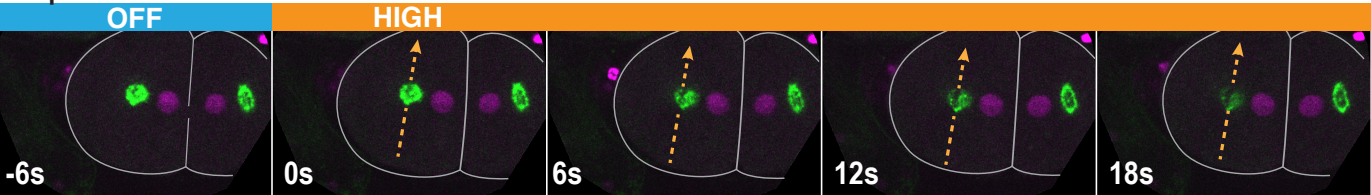

$\mathbf{G}$

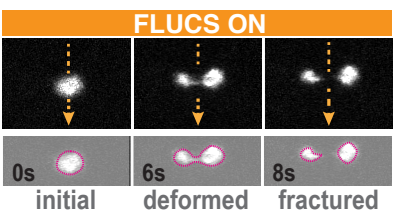

$\Theta \quad \Longleftrightarrow$

initial length final length
Fracture probablity $=$

Frequency of FLUCS events ending with PCM separation

Deformation rate $=$ final-initial length time
$\mathrm{H}$

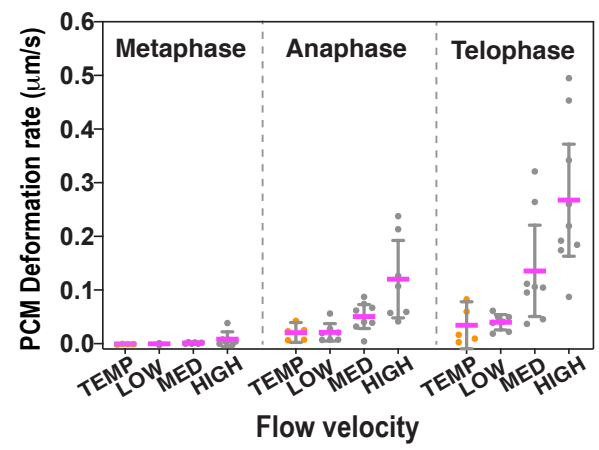

OFF

TEMP control =

$10 \mathrm{kHz}, 40 \mathrm{~mW}$

bidirectional scan;

no flow, only heat

I
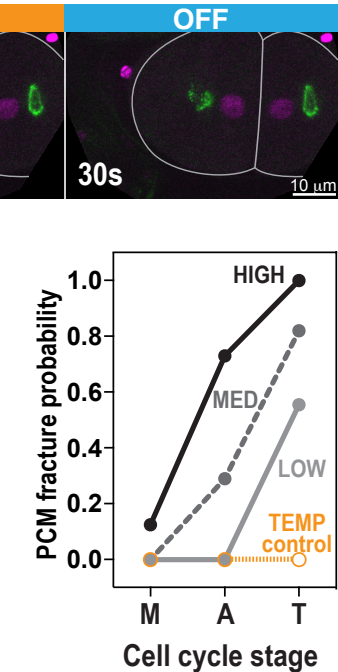


\section{Mittasch et al. Figure 2}

\section{A gpr-1/2(RNAi) --- reduced cortical pulling force}

Metaphase
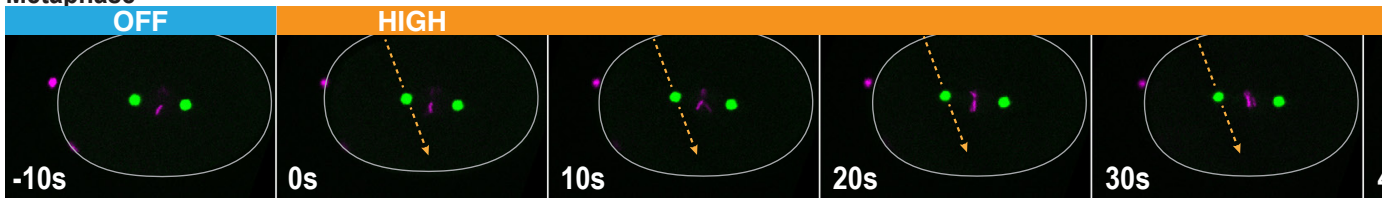

mMaple::SPD-5 mCherry::H2B

Anaphase

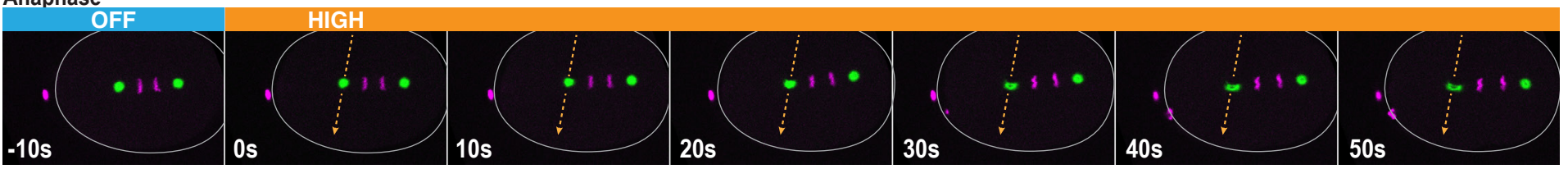

Telophase

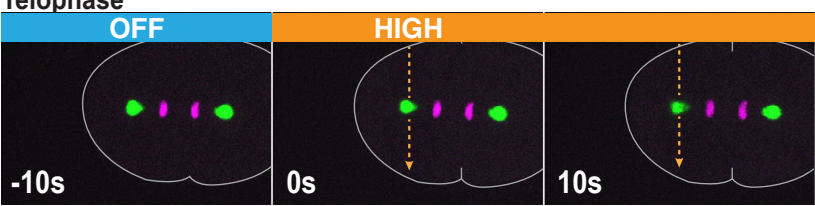

$20 s$

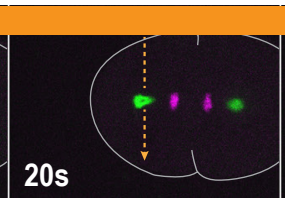

$30 \mathrm{~s}$

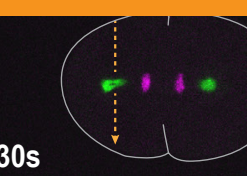

$40 s$

50 s

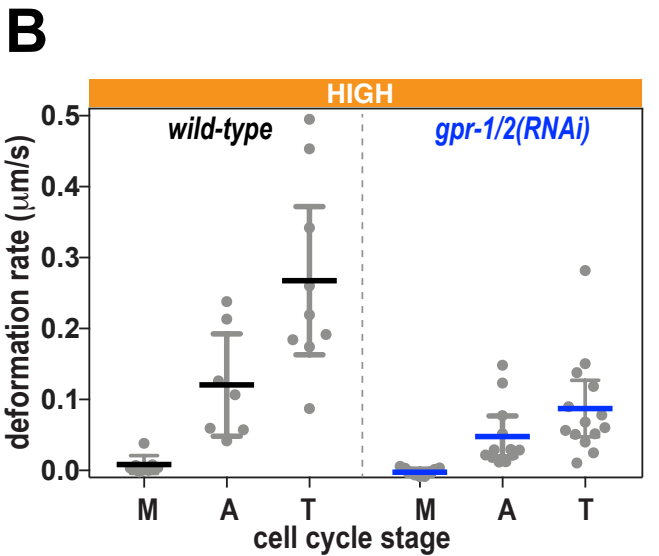

E

gpr-1/2(RNAi)

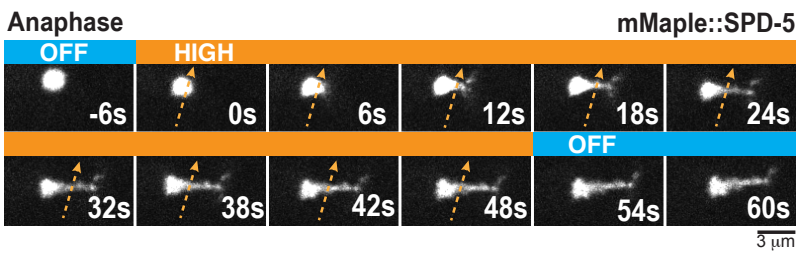

D

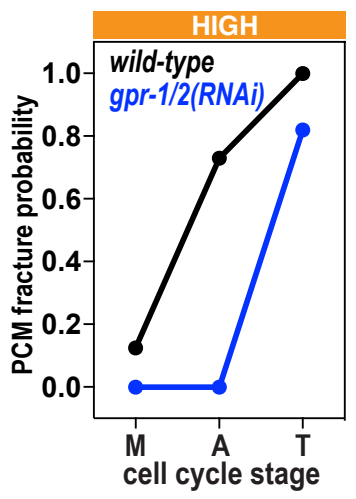

+ nocodazole

Telophase
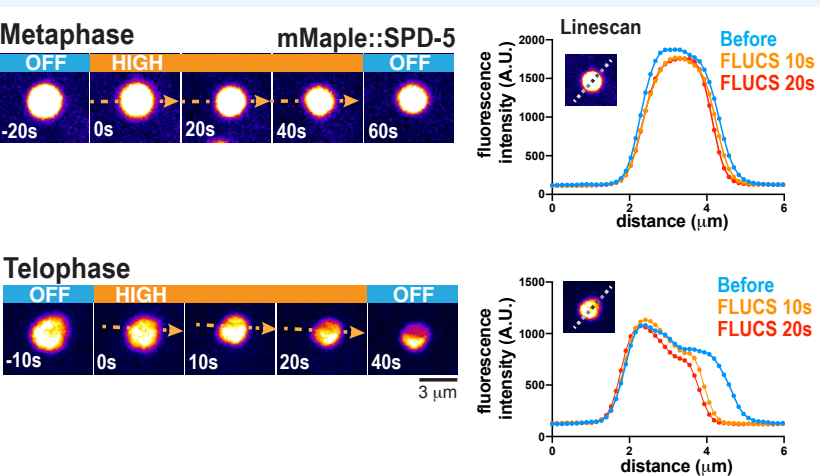

F
gpr-1/2(RNAi)
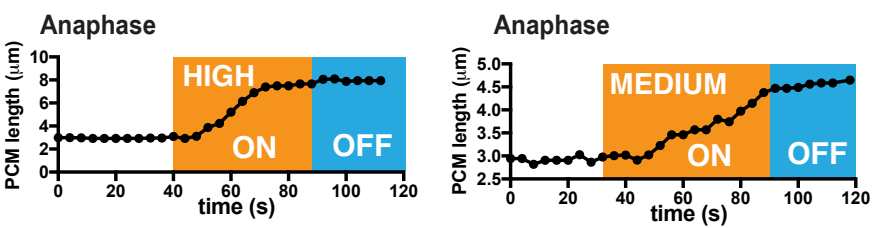
Mittasch et al. Figure 3
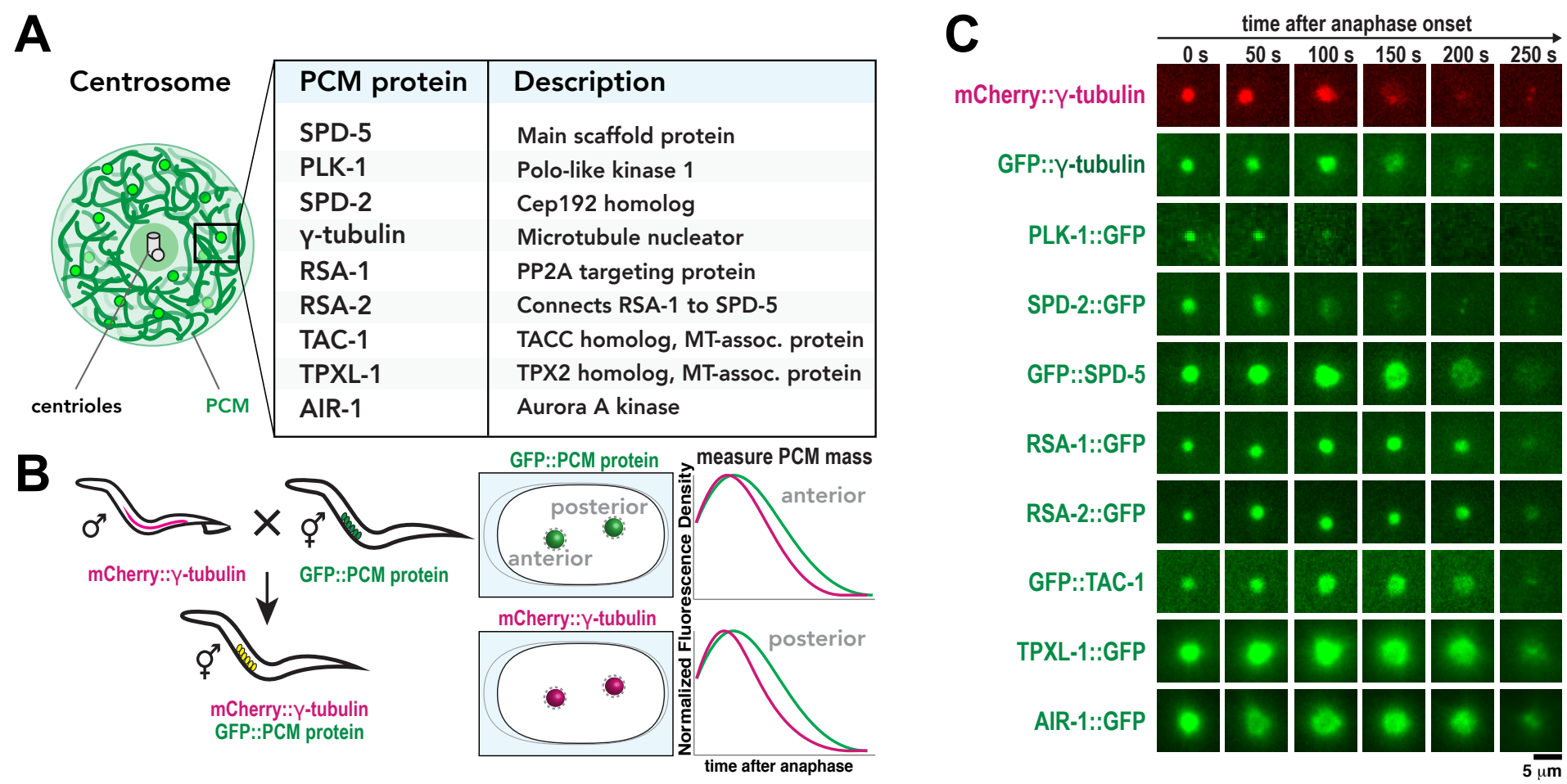

D Disassembly profiles for anterior PCM proteins
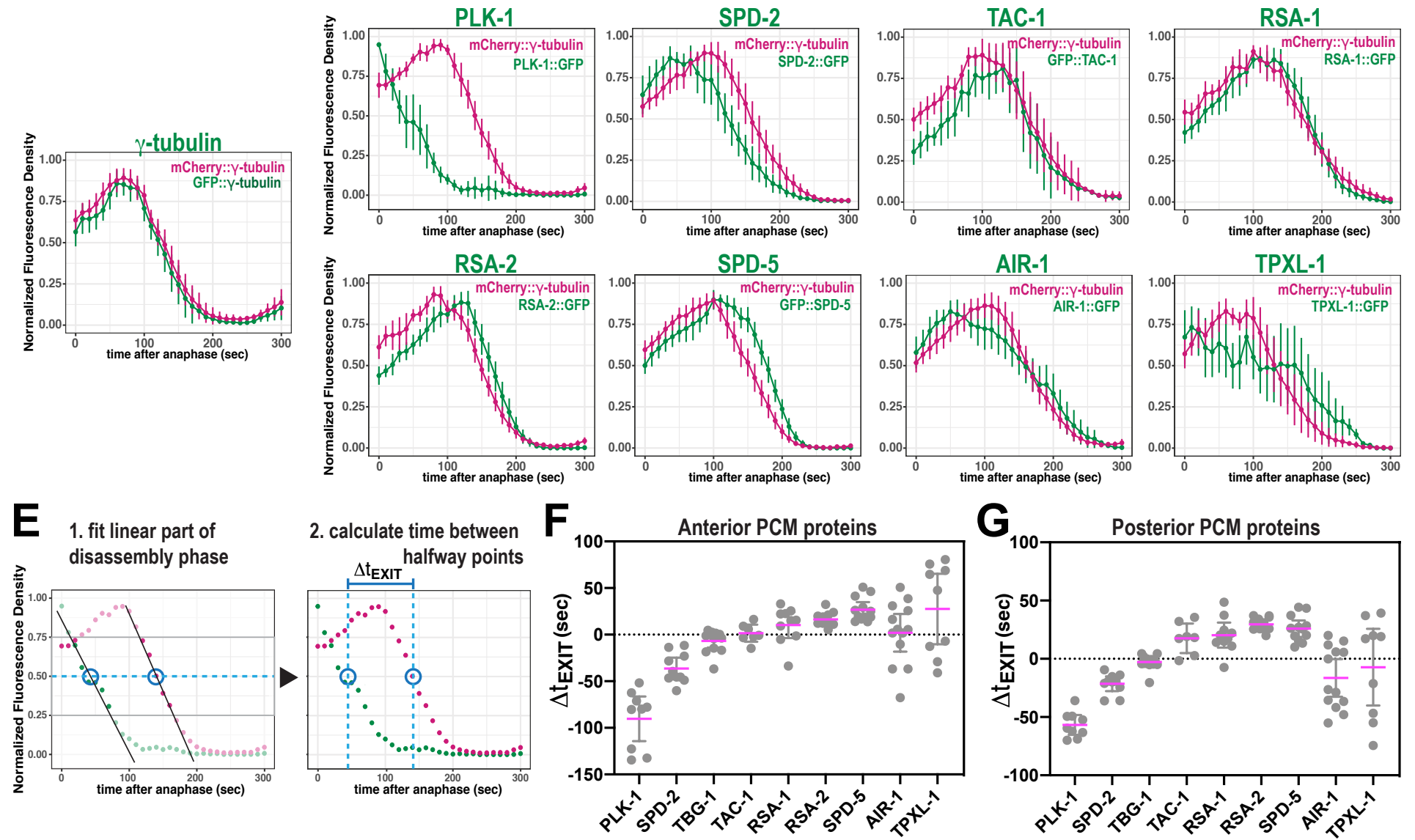
A Inhibition of PLK-1 in Metaphase

wild-type

mMaple::SPD-5

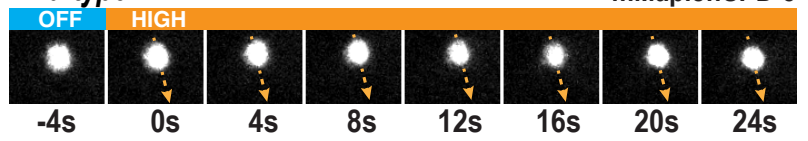

$+\mathrm{Bl}-2536$

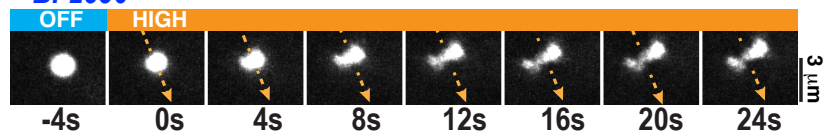

B

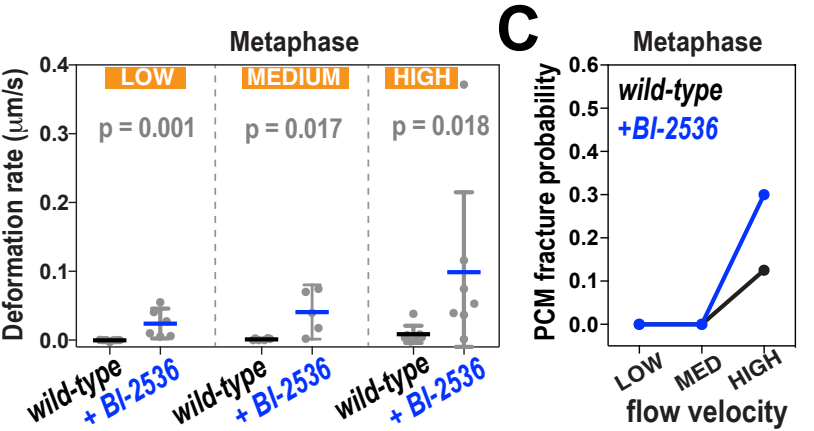

D PCM stability in Metaphase-arrested embryos

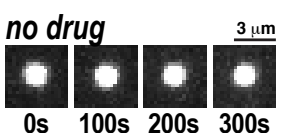

$+B \mathrm{I}-2536$
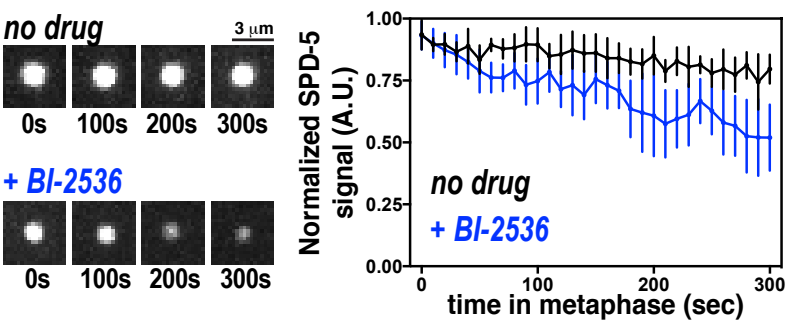

E spd-2(or188ts) in Metaphase

after up-shift to $25^{\circ} \mathrm{C}$

GFP::SPD-5
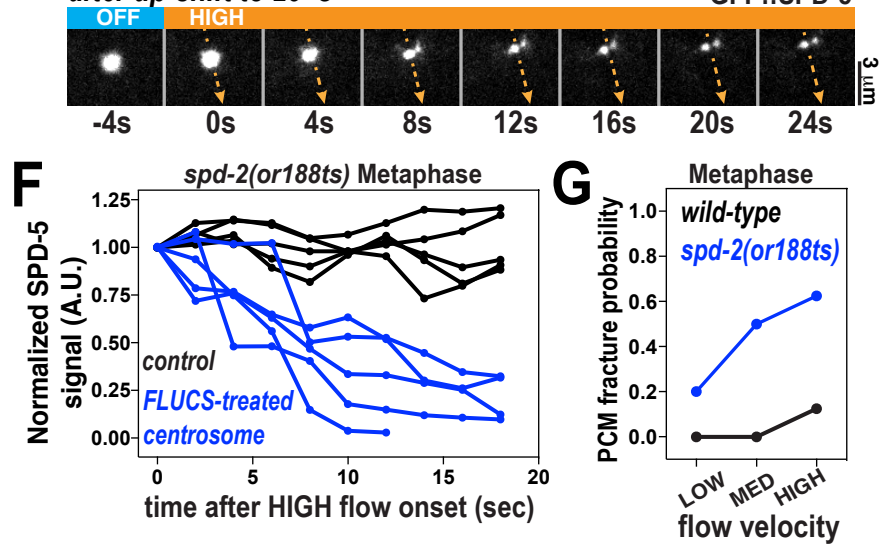

\section{H PCM disassembly in Anaphase}
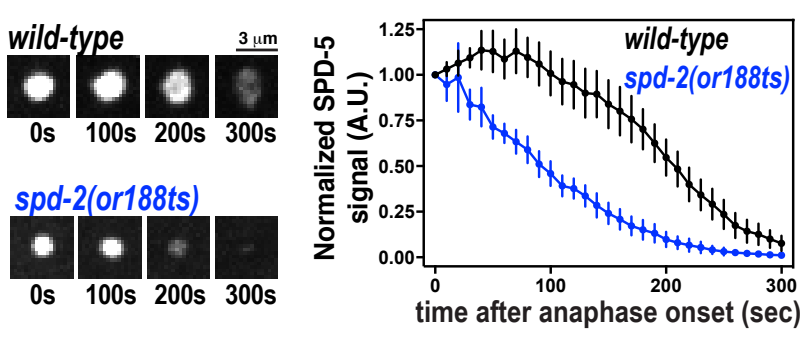
Mittasch et al. Figure 5

A In vitro PCM disassembly assay

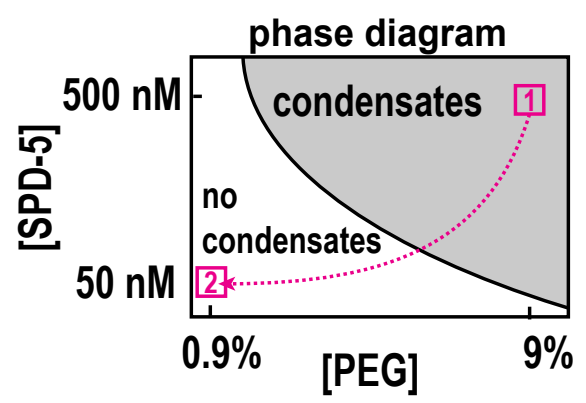

C
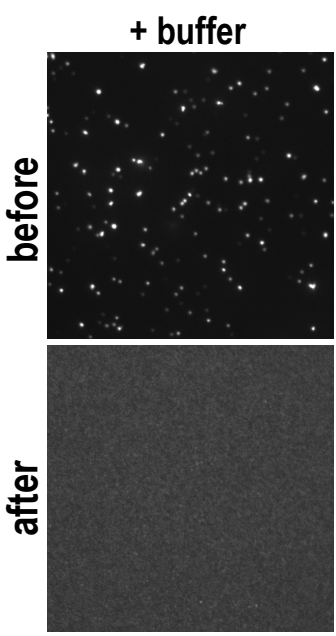

1. before

SPD-5::RFP

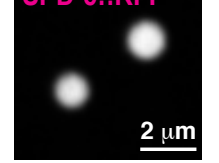

2. after

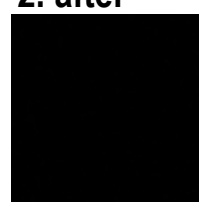

500 nM SPD-5::RFP

+ PLK-1 1 KD
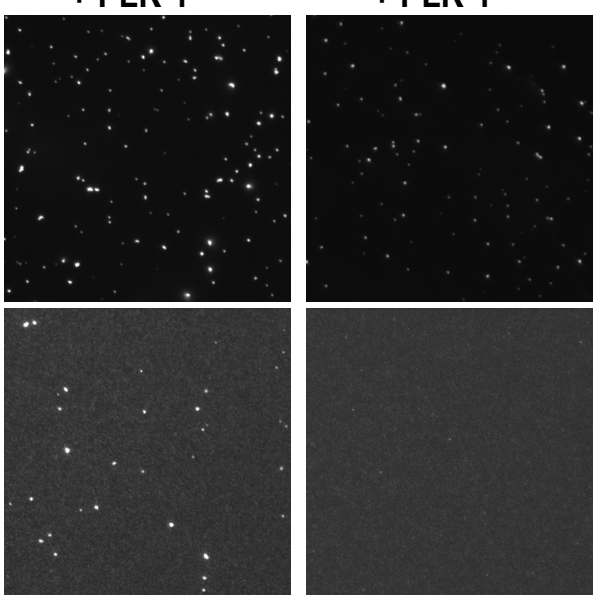

B

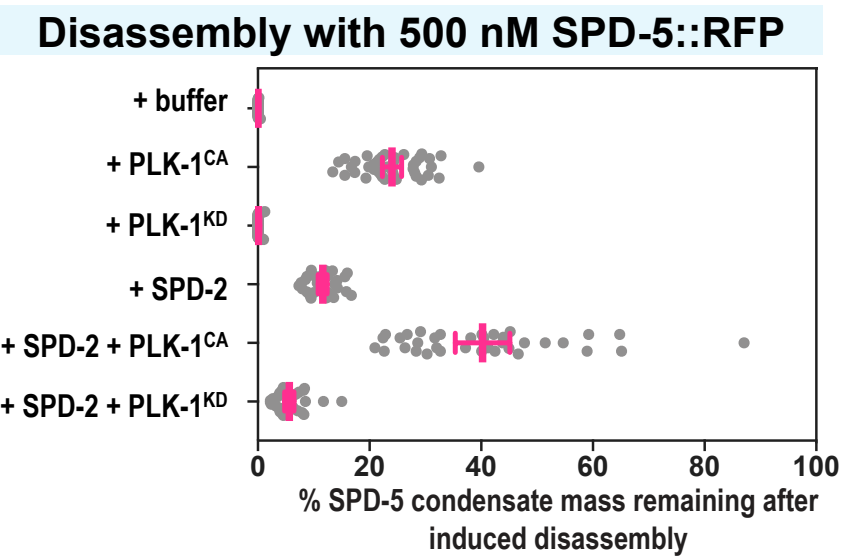

+ SPD-2

+ SPD-2 + PLK-1 ${ }^{\mathrm{CA}}+\mathrm{SPD}-2+\mathrm{PLK}-1^{\mathrm{KD}}$
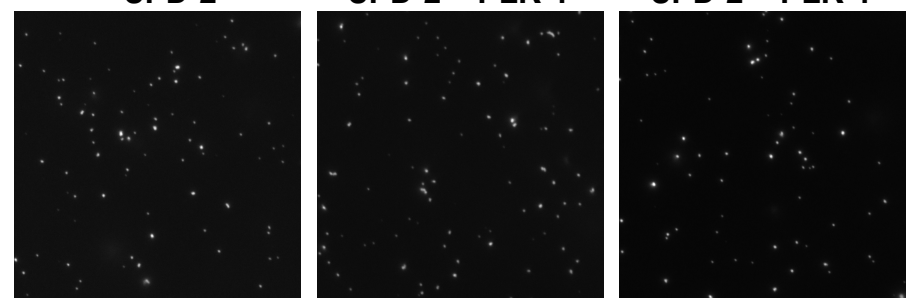
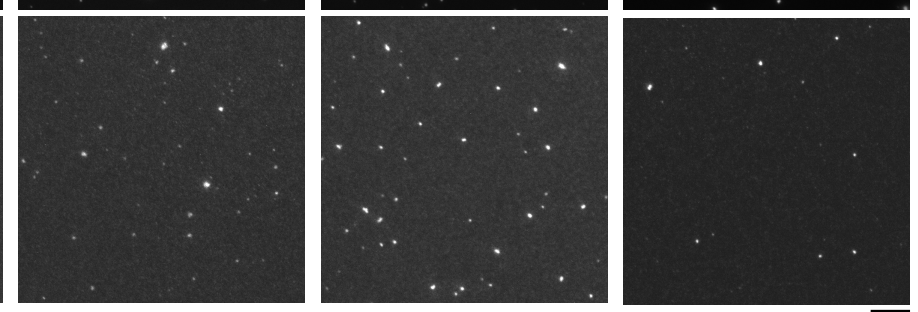

$10 \overline{\mu \mathrm{m}}$ 
bioRxiv preprint doi: https://doi.org/10.1101/866434; this version posted December 5,2019 . The copyright holder for this preprint (which was not certified by peer review) is the author/funder, who has granted bioRxiv a license to display the preprint in perpetuity. It is made available under aCC-BY-NC-ND 4.0 International license.

Mittasch et al. Figure 6

A Inhibition of PP2A in Anaphase

wild-type

mMaple::SPD-5
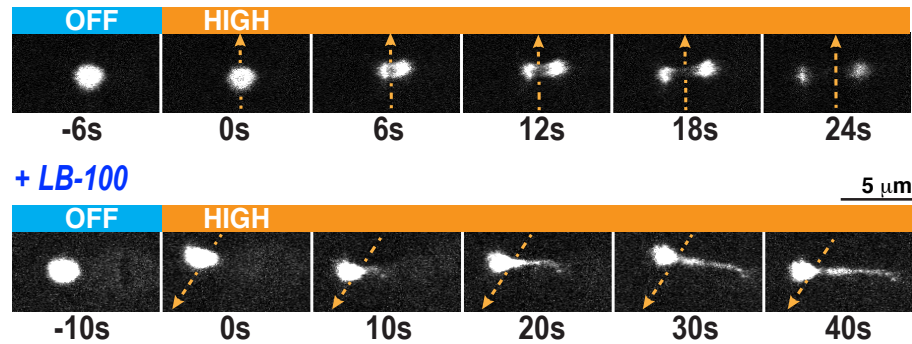

B
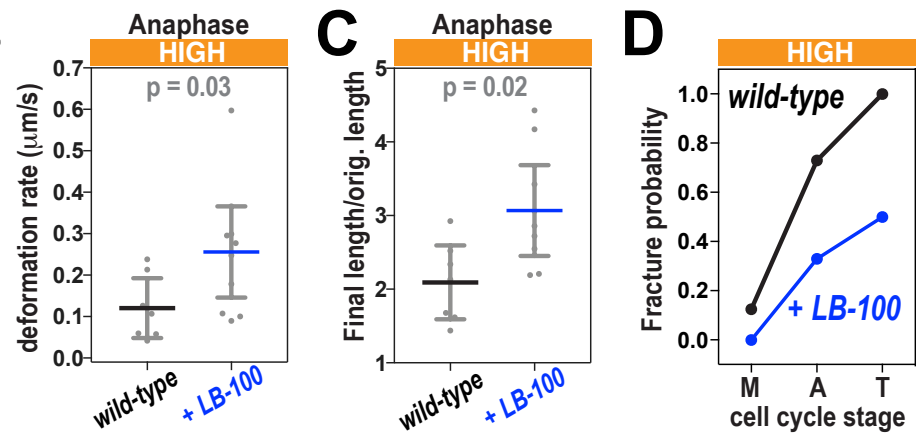

E PCM disassembly in Anaphase
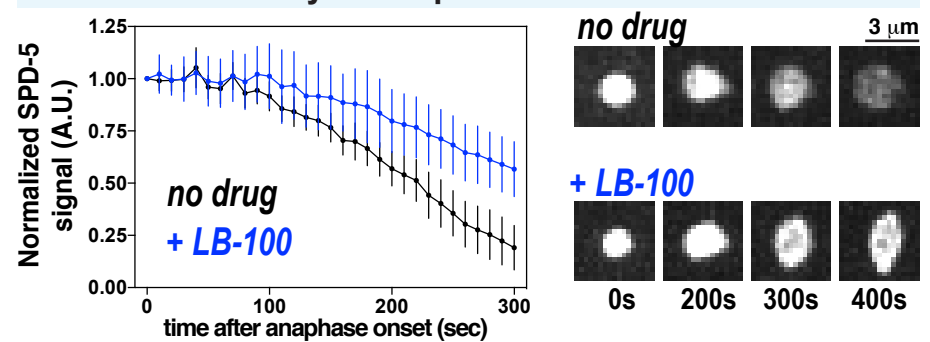

\section{$+L B-100$}

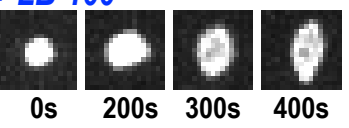

F PP2A localization during PCM disassembly
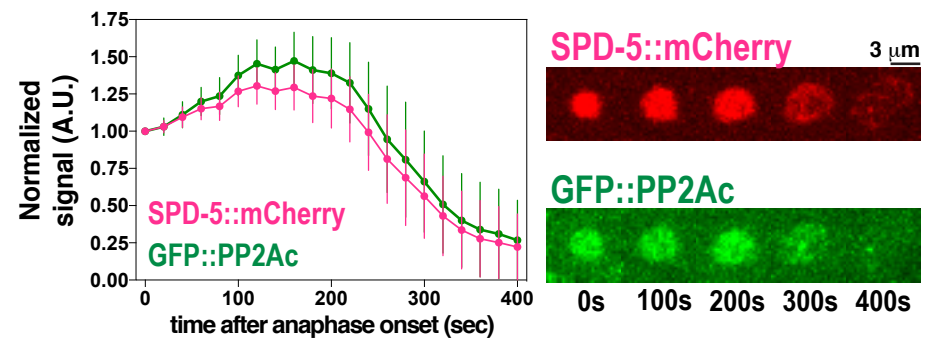

GFP::PP2AC

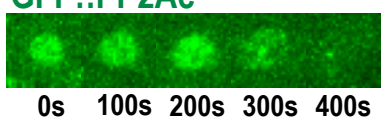


Mittasch et al. Figure 7

A PCM mechanical properties
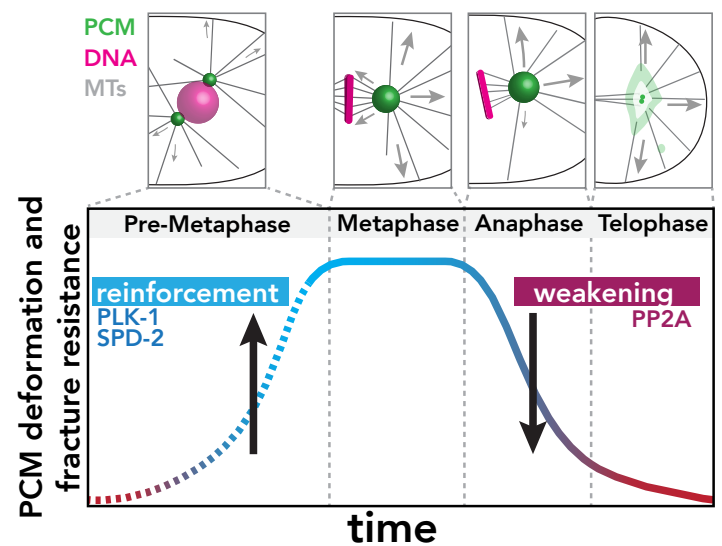

C

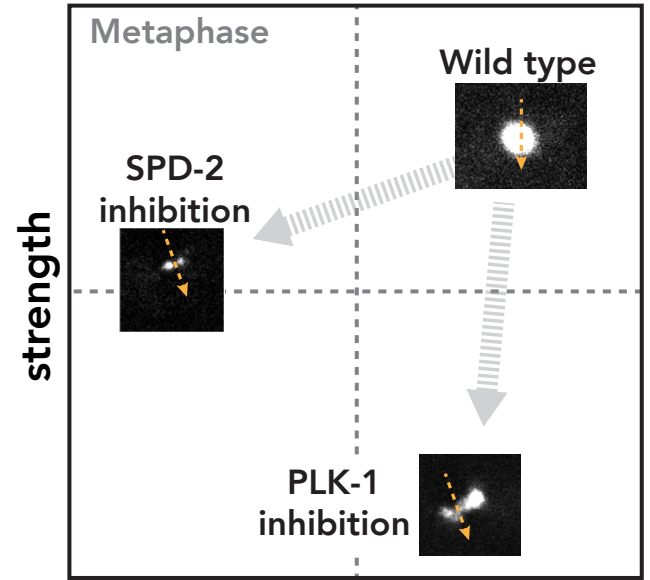

ductility
B

PLK-1(Polo Kinase)

SPD-2 (Cep192)

$\gamma$-tubulin

TAC-1 (TACC)

SPD-5

RSA-2

RSA-1

PP2Ac

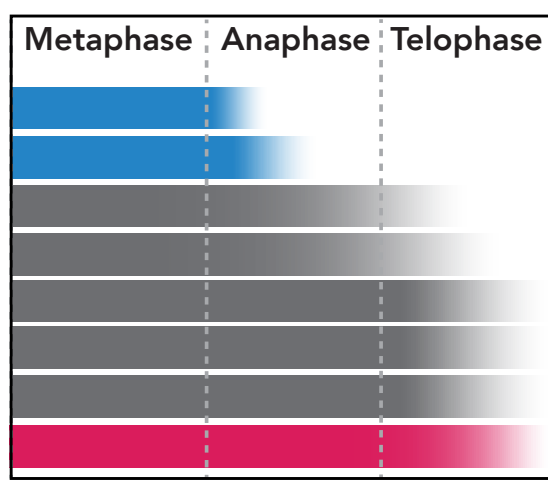

PCM protein levels

D Natural PCM disassembly

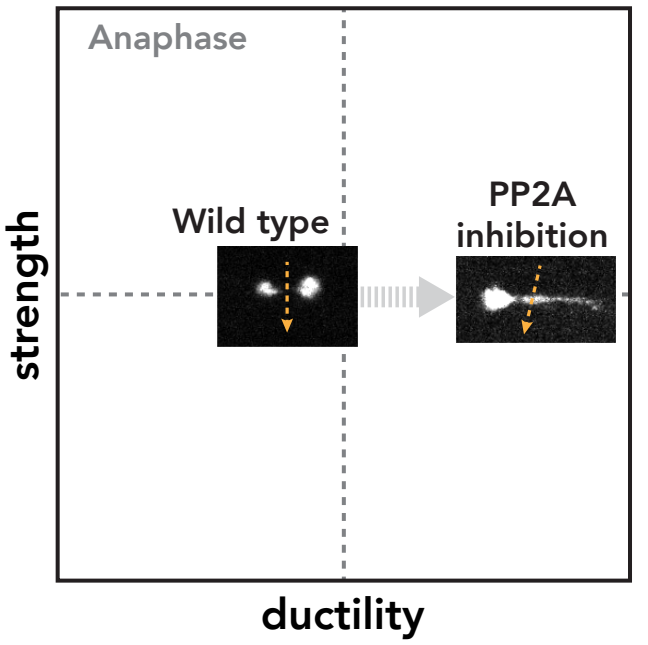

time

ductility

Absent

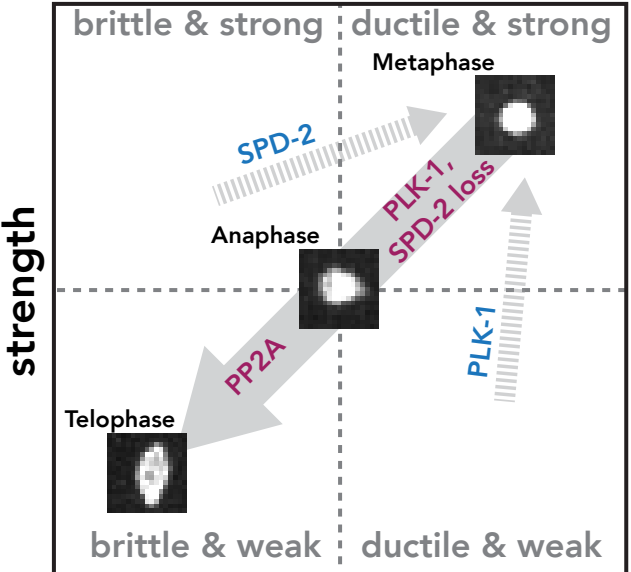


A

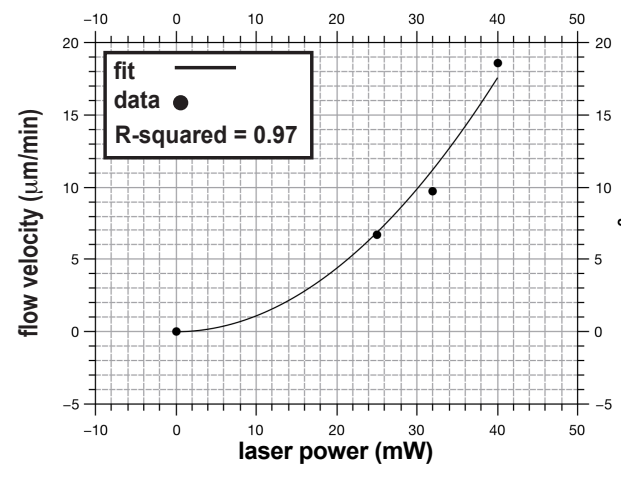

B

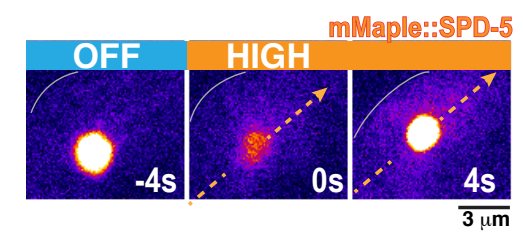

Prometaphase

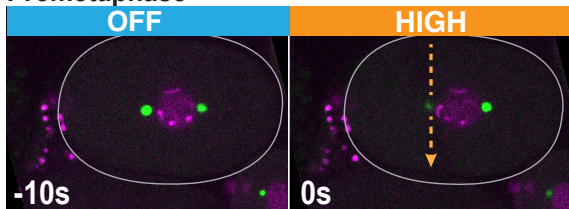

$-10 s$ Os
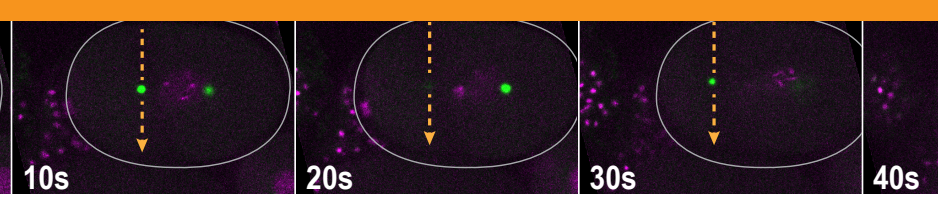

mMaple::SPD-5 mCherry::H2B

\section{Continuous FLUCS during metaphase-anaphase transition}

Metaphase-Anaphase
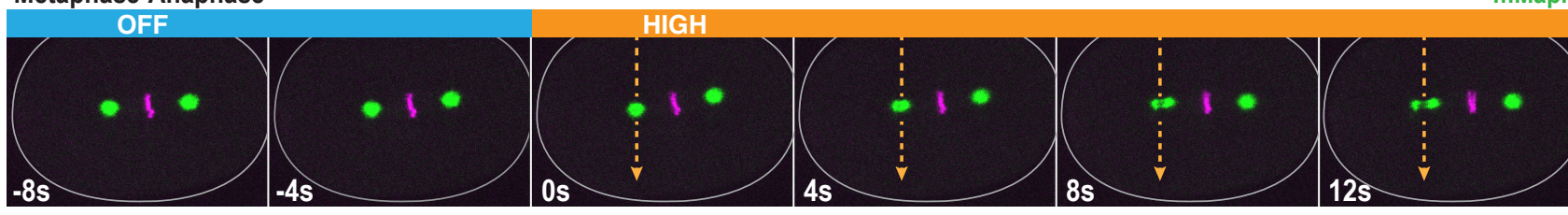

mMaple::SPD-5 mCherry::H2B

\section{E $10 \mathrm{kHz}$, bidirectional laser scan---no flow, only heat}

Anaphase-Telophase

mMaple::SPD-5 mCherry::H2B
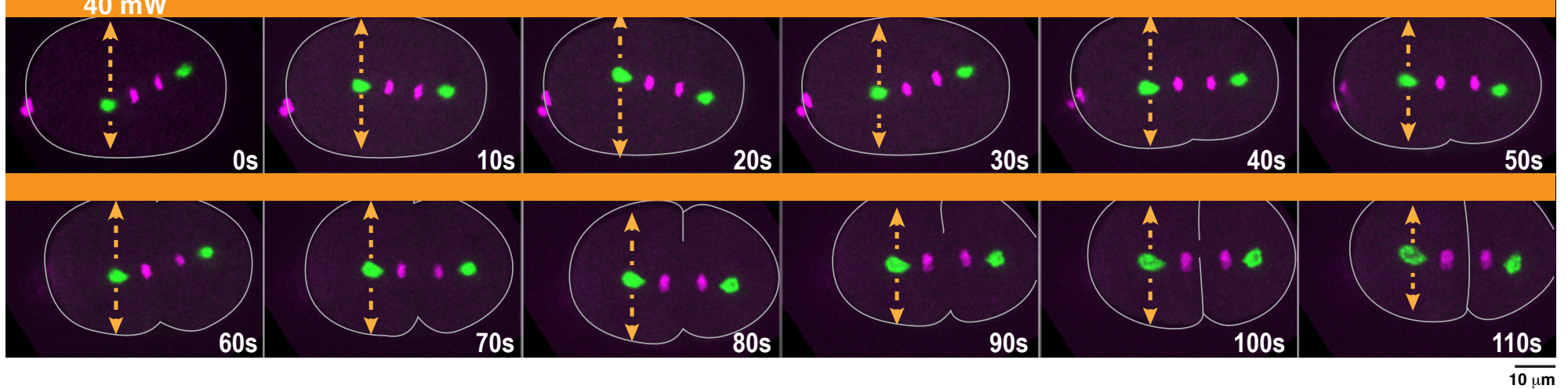

\section{F Embryo survival after FLUCS in one-cell stage}
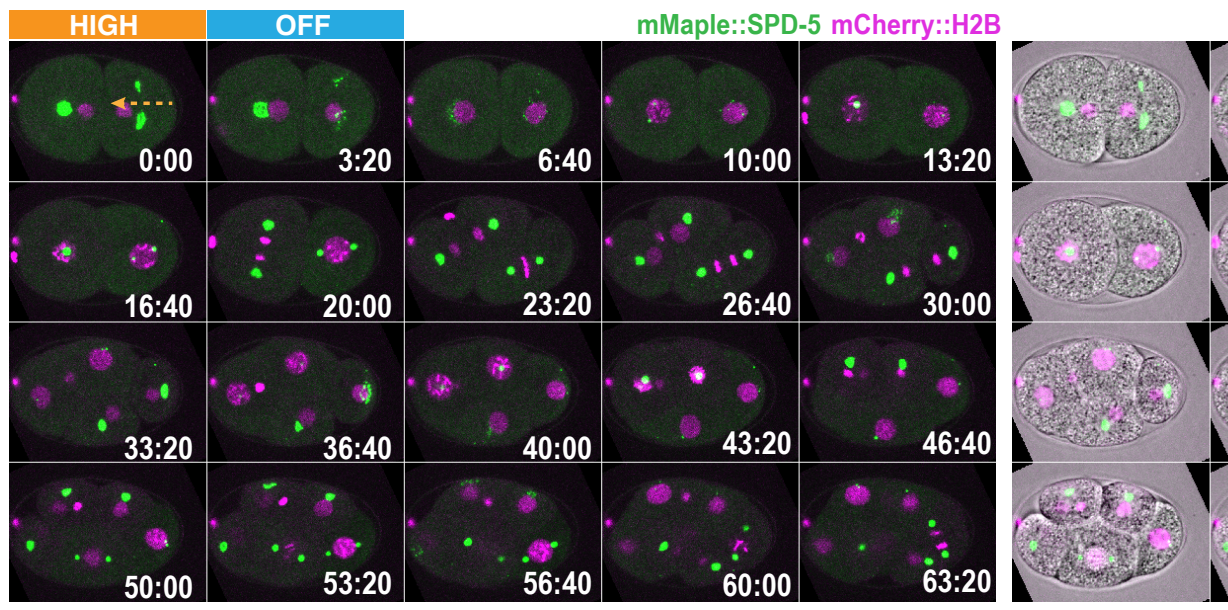

Brightfield images

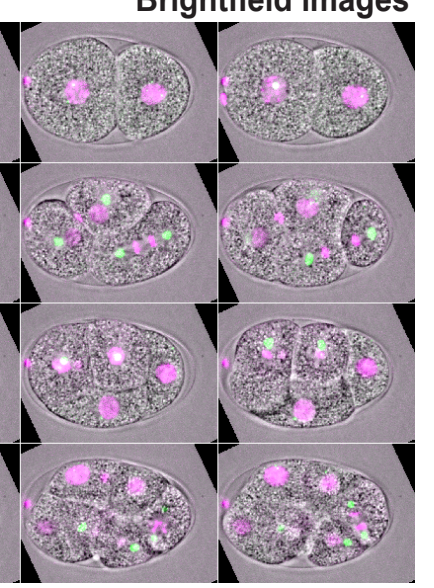


bioRxiv preprint doi: https://doi.org/10.1101/866434; this version posted December 5, 2019. The copyright holder for this preprint (which was not certified by peer review) is the author/funder, who has granted bioRxiv a license to display the preprint in perpetuity. It is made available under aCC-BY-NC-ND 4.0 International license.

Mittasch et al. Figure S2

A

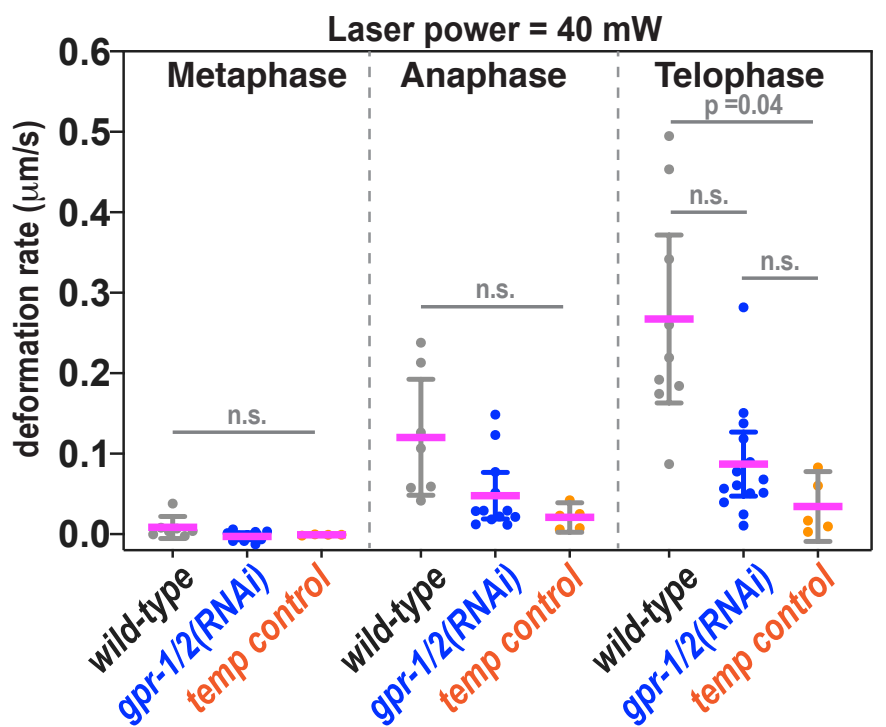

B csnk-1RNAi) --- increased pulling forces throughout the cell cycle
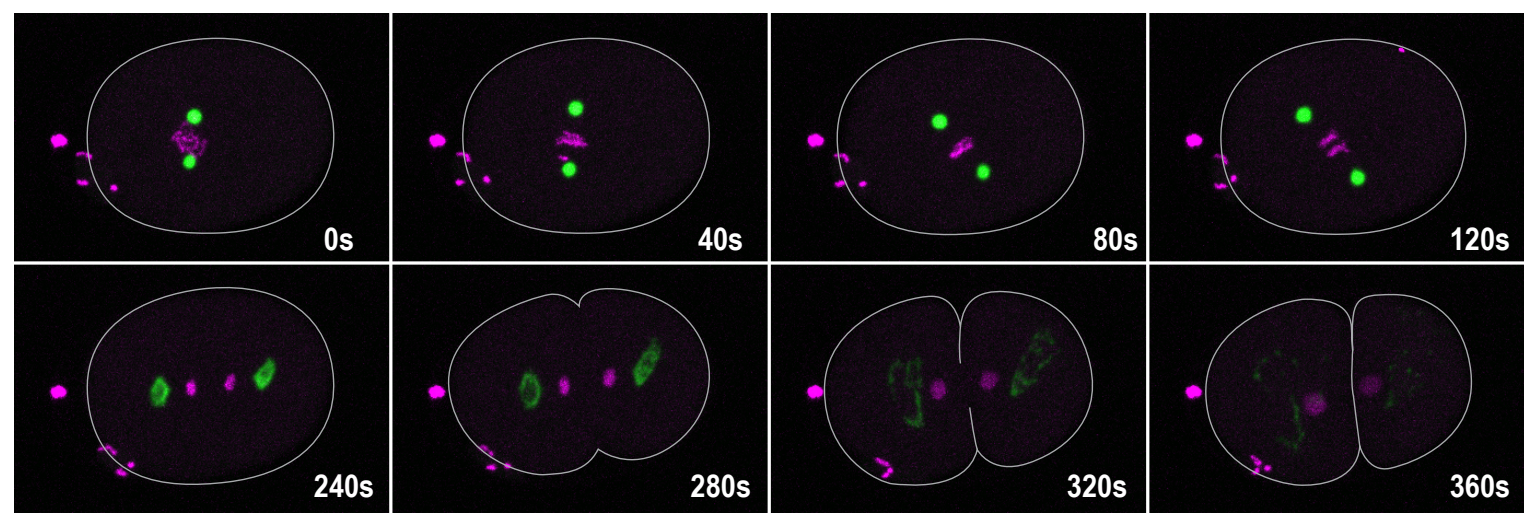

mMaple::SPD-5 mCherry::H2B
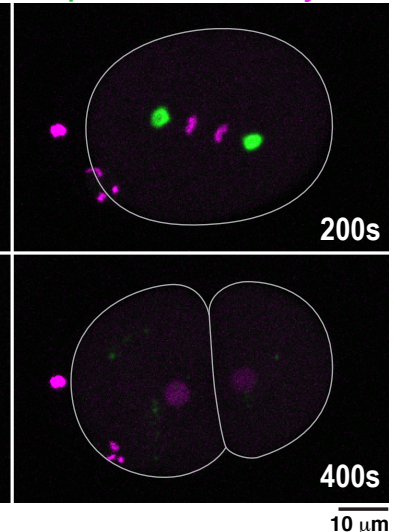


\section{Mittasch et al. Figure S3}

\section{Disassembly profiles for posterior PCM proteins}
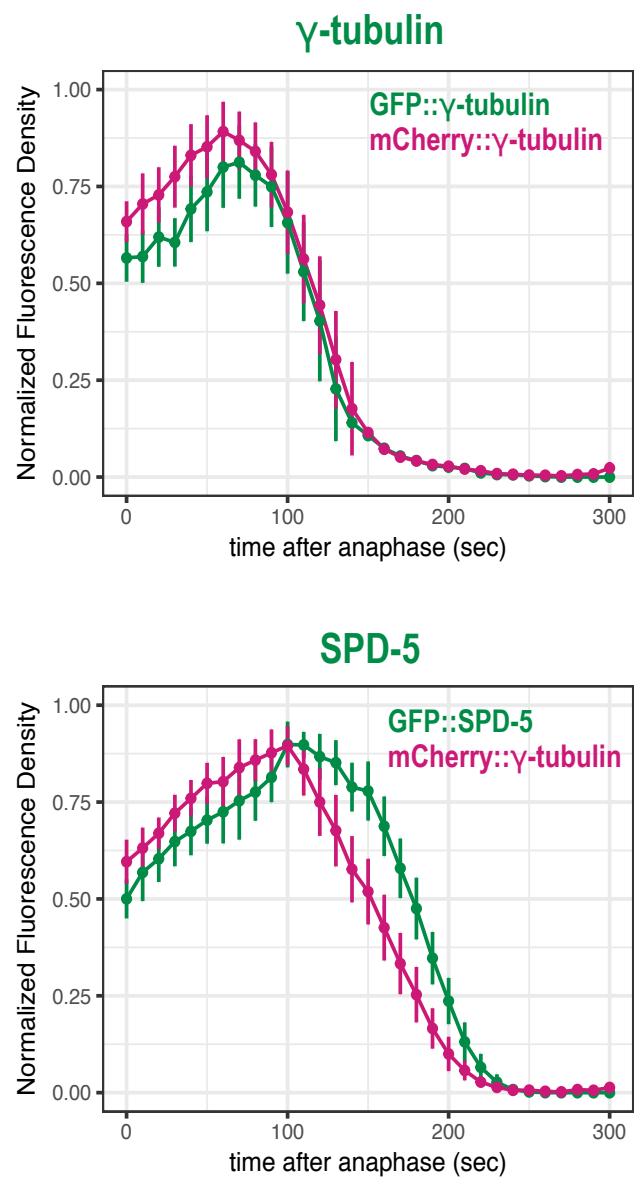

TAC-1

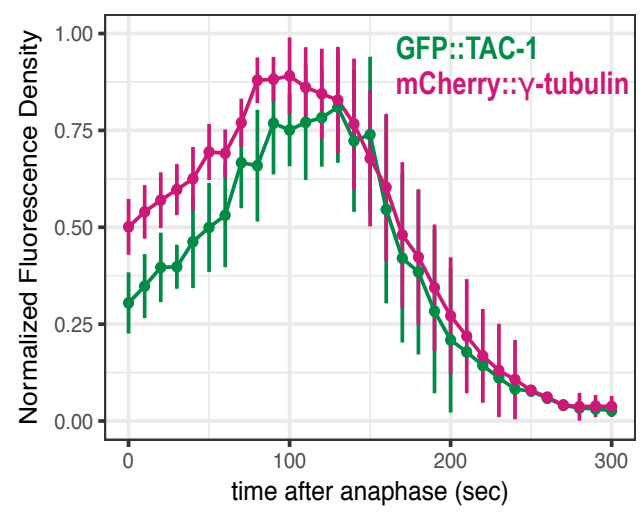

PLK-1

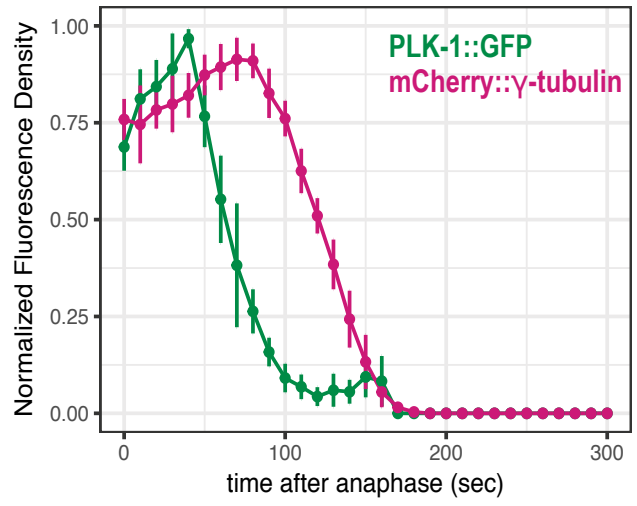

RSA-1

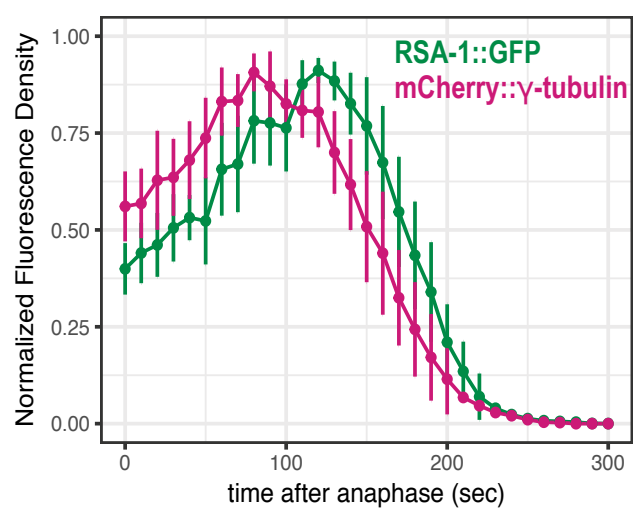

TPXL-1

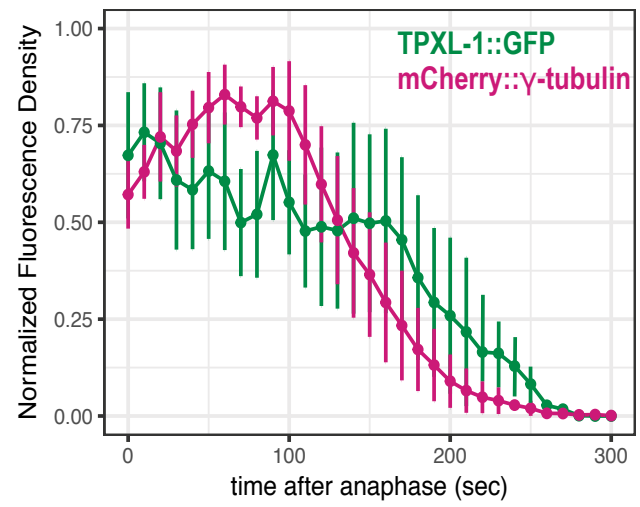

SPD-2

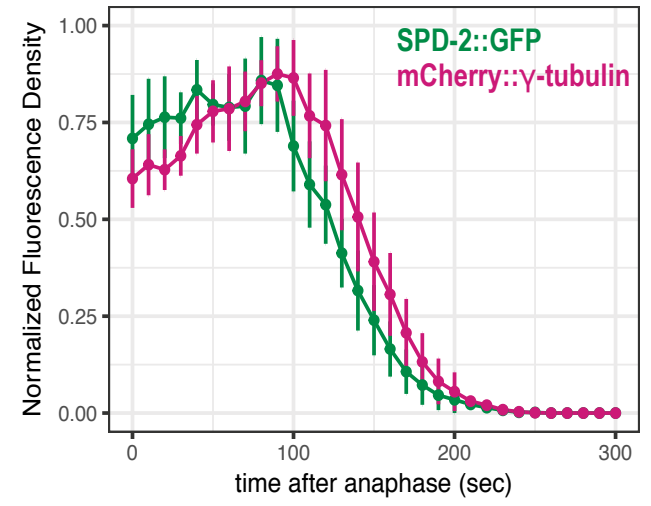

RSA-2

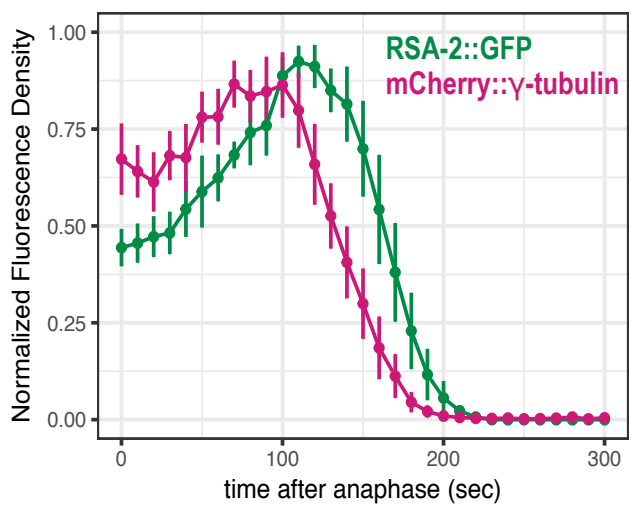

AIR-1

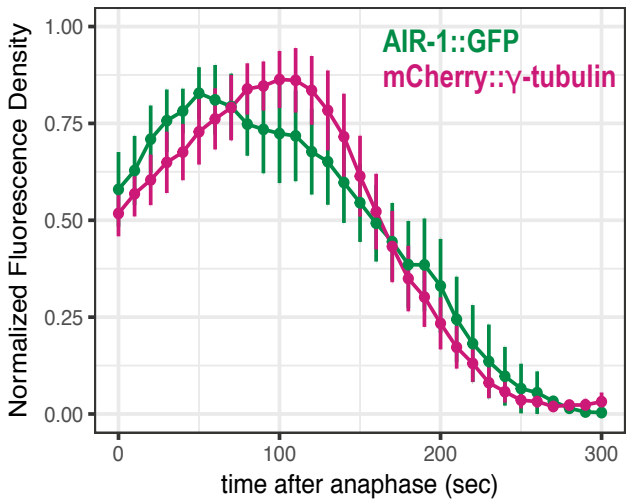




\section{Mittasch et al. Figure S4}
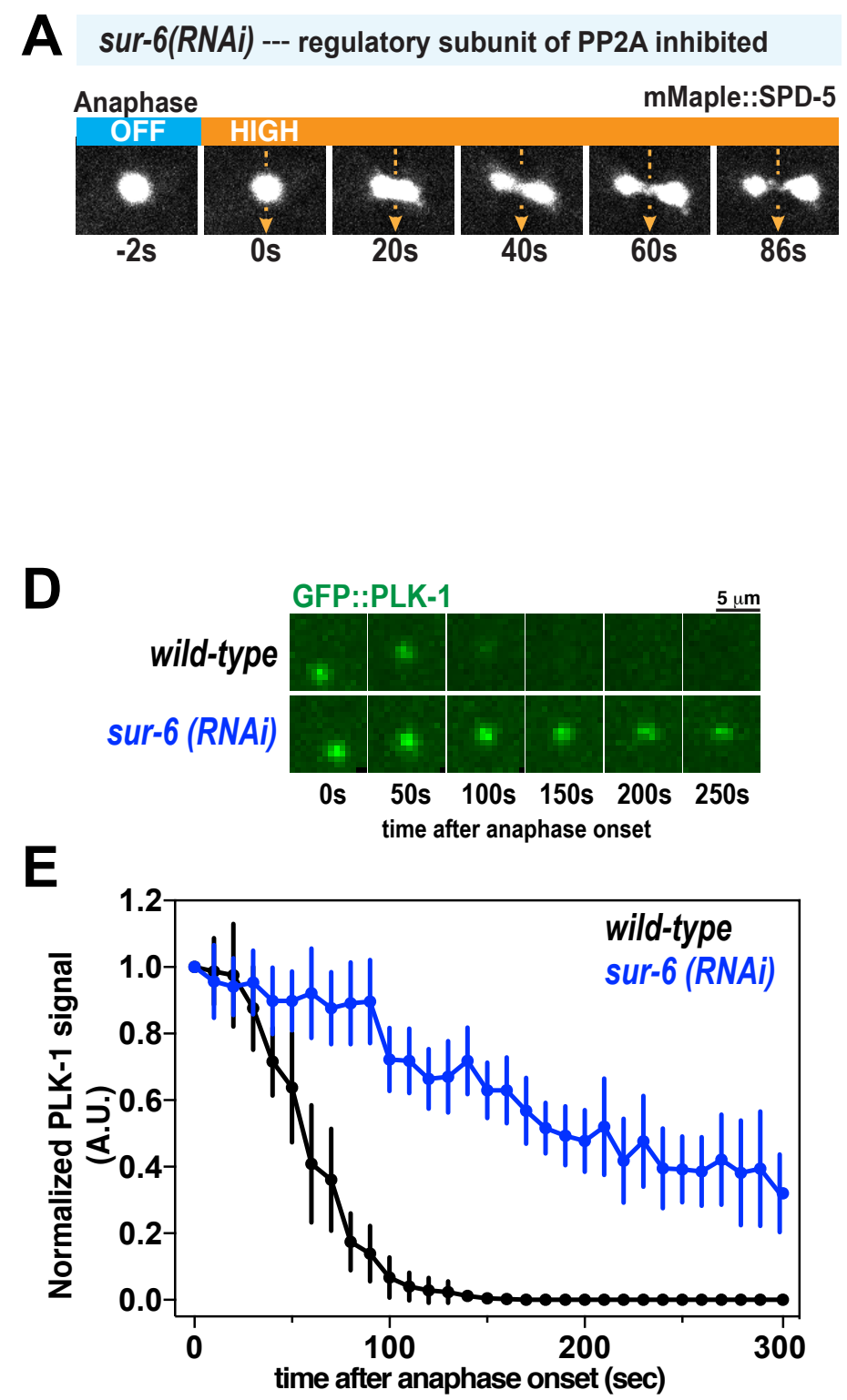
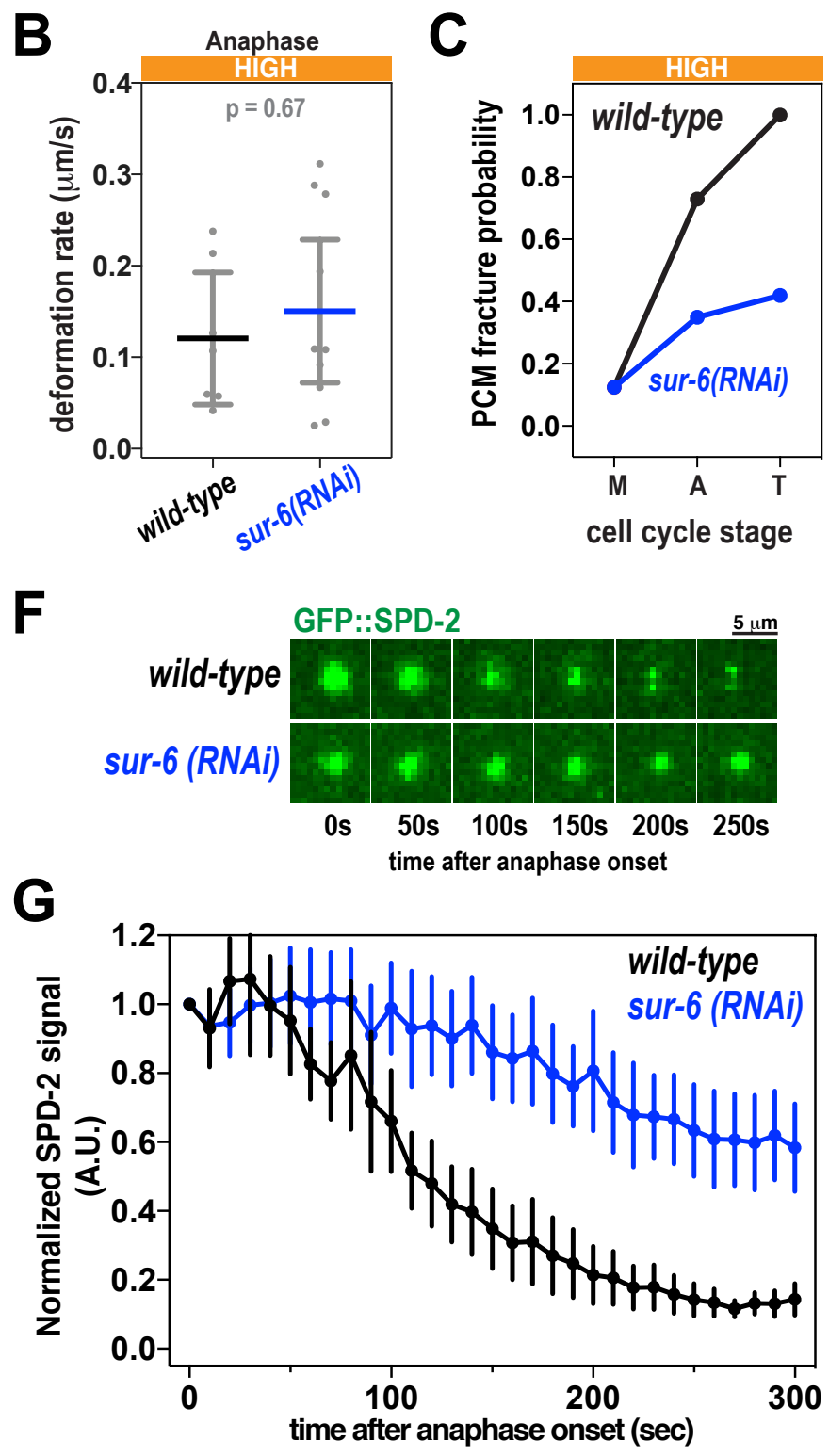